\title{
Duo: a general program for calculating spectra of diatomic molecules ${ }^{\text {th }}$
}

\author{
Sergei N. Yurchenko,,*, Lorenzo Lodi ${ }^{\mathrm{a}}$, Jonathan Tennyson ${ }^{\mathrm{a}}$, Andrey V. \\ Stolyarov ${ }^{\mathrm{b}}$ \\ ${ }^{a}$ Department of Physics $\mathcal{E}$ Astronomy, University College London, Gower Street, London \\ WC1E 6BT, United Kingdom \\ ${ }^{b}$ Department of Chemistry, Lomonosov Moscow State University, Leninskiye gory 1/3, 119992 \\ Moscow, Russia
}

\begin{abstract}
Duo is a general, user-friendly program for computing rotational, rovibrational and rovibronic spectra of diatomic molecules. Duo solves the Schrödinger equation for the motion of the nuclei not only for the simple case of uncoupled, isolated electronic states (typical for the ground state of closed-shell diatomics) but also for the general case of an arbitrary number and type of couplings between electronic states (typical for open-shell diatomics and excited states). Possible couplings include spin-orbit, angular momenta, spin-rotational and spin-spin. Corrections due to non-adiabatic effects can be accounted for by introducing the relevant couplings using so-called Born-Oppenheimer breakdown curves.

Duo requires user-specified potential energy curves and, if relevant, dipole moment, coupling and correction curves. From these it computes energy levels, line positions and line intensities. Several analytic forms plus interpolation and extrapolation options are available for representation of the curves. Duo can refine potential energy and coupling curves to best reproduce reference data such as experimental energy levels or line positions. Duo is provided as a Fortran 2003 program and has been tested under a variety of operating systems.
\end{abstract}

Keywords: diatomics, spectroscopy, one-dimensional Schrödinger equation, excited electronic states, intramolecular perturbation, coupled-channel radial equations, transition probabilities, intensities

\footnotetext{
This paper and its associated computer program are available via the Computer Physics Communication homepage on ScienceDirect

*Corresponding author

Email addresses: s.yurchenko@ucl.ac.uk (Sergei N. Yurchenko), 1.1odi@ucl .ac.uk (Lorenzo Lodi), j.tennyson@ucl . ac .uk(Jonathan Tennyson), avstol@phys . chem.msu.ru (Andrey V. Stolyarov)
} 


\section{Program summary}

Program title: Duo

Catalogue number:

Program summary URL:

Program obtainable from: CPC Program Library, Queen's University, Belfast, N. Ireland

Licensing provisions: Standard CPC licence.

No. of lines in distributed program, including test data, etc.: 160049

No. of bytes in distributed program, including test data, etc.: 13957785

Distribution format: tar.gz

Programming language: Fortran 2003.

Computer: Any personal computer.

Operating system: Linux, Windows, Mac OS.

Has the code been vectorized or parallelized?: Parallelized.

Memory required to execute: case dependent, typically $<10 \mathrm{MB}$

Nature of physical problem: Solving the Schrödinger equation for the nuclear motion of a diatomic molecule with an arbitrary number and type of couplings between electronic states.

Solution method: Solution of the uncoupled problem first, then basis set truncation and solution of the coupled problem. A line list can be computed if a dipole moment function is provided. The potential energy and other curves can be empirically refined by fitting to experimental energies or frequencies, when provided. Restrictions on the complexity of the problem: The current version is restricted to bound states of the system.

Unusual features of the program: User supplied curves for all objects (potential energies, spin-orbit and other couplings, dipole moment etc) as analytic functions or tabulated on a grid is a program requirement.

Typical running time: Case dependent. The test runs provided take seconds or a few minutes on a normal PC.

\section{Introduction}

Within the Born-Oppenheimer or adiabatic approximation [1] the rotationalvibrational (rovibrational) energy levels of a diatomic molecule with nuclei $a$ and $b$ and in a ${ }^{1} \Sigma^{ \pm}$electronic state are given by the solution of the one-dimensional Schrödinger equation:

$$
-\frac{\hbar^{2}}{2 \mu} \frac{\mathrm{d}^{2}}{\mathrm{~d} r^{2}} \psi_{v J}(r)+\left[V_{\text {state }}(r)+\frac{J(J+1)}{2 \mu r^{2}}\right] \psi_{v J}(r)=E_{v J} \psi_{v J}(r),
$$


where $\mu^{-1}=M_{a}{ }^{-1}+M_{b}{ }^{-1}$ is the reduced mass of the molecule and $M_{a}$ and $M_{b}$ are the (nuclear) masses of atoms $a$ and $b$, respectively. $V_{\text {state }}(r)$ is the potential energy curve (PEC) for the electronic state under study, $J$ is the total angular momentum of the molecule and $v=0,1, \ldots$ is the vibrational quantum number. The solution of this one-dimensional Schrödinger equation is a well-studied mathematical problem [2, 3] for which many efficient numerical methods are available [4-19]; the most popular of them is probably the iterative "shooting" Cooley-Numerov [20-22] method which is notably used in the program Level due to Le Roy [23].

As well as the 'direct' problem of solving the Schrödinger equation for a given PEC, also of great interest is the corresponding inverse problem [24, 25], that is the task of determining the potential $V_{\text {state }}(r)$ which leads to a given set of energy levels $E_{v J}$, typically obtained from experiment. A traditional way of performing this task approximately is to use the semi-classical Rydberg-Klein-Rees (RKR) method [24]; a more precise strategy called inverse perturbation analysis (IPA) has been suggested by Kosman and Hinze $[25,26]$ and a program implementing this approach was presented by Pashov et al [27]. A different, grid-based fitting strategy has been recently suggested by Szidarovszky and Császár [28]. The program DPotFit [29], a companion to Le Roy's LeVEL, also provides this functionality for isolated states of closed shell diatomics. Indeed, for single potential problems there is an extensive literature on the determination of potential curves from experimental data; in this context we particularly note the work of Coxon and Hajigeorgiou [30-32] and Le Roy and co-workers [33-36].

When the diatomic molecule has a more complex electronic structure (i.e., the electronic term is not ${ }^{1} \Sigma$ ) the situation is more complicated, as interactions between the various terms are present and it is not possible to treat each electronic state in isolation. Although there are a growing number of studies treating coupled electronic states, for example see Refs. [37-44], there appears to be no general program available for solving the coupled problem, the closest being a general coupled-state program due to Hutson [45]. We have therefore developed a new computer program, Duo, particularly to deal with such complex cases.

Duo is a flexible, user-friendly program written in Fortran 2003 and capable of solving both the direct and the inverse problem for a general diatomic molecule with an arbitrary number and type of couplings between electronic states, including spin-orbit, electronic-rotational, spin-rotational and spin-spin couplings. Duo also has auxiliary capabilities such as interpolating and extrapolating curves and calculating lists of line positions and line intensities (so-called line lists). Duo is currently being used as part of the ExoMol project [46], whose aim is to generate high-temperature spectra for all molecules likely to be observable in exoplanet atmospheres in the foreseeable future. Completed studies based on the use of Duo include ones on $\mathrm{AlO}$ [47, 48], $\mathrm{ScH}$ [49], $\mathrm{CaO}$ [50] and VO [51]. Our methodology is the subject of a very recent topical review [52]. 
This paper is organised as follows. In Section 2 we review the theory and the basic equations used by Duo to solve the coupled nuclear motion problem for diatomics. In Section 3 we discuss the calculation of molecular line intensities and line lists. Section 4 is devoted to the inverse problem, i.e. to the refinement ('fitting') of potential and coupling curves so that they reproduce a set of reference energy levels or line positions. Section 5 reviews the functional forms implemented for the various curves. In Section 6 the program structure is explained. Finally, we draw our conclusions in Section 7. Technical details on program usage such as detailed explanations of the program options and sample inputs are reported in a separate user's manual.

\section{Method of solution}

After separating out the centre-of-mass motion and having introduced a bodyfixed set of Cartesian axes with origin at the centre of nuclear mass and with the $z$ axis along the internuclear direction the non-relativistic Hamiltonian of a diatomic molecule can be written as [53-57]:

$$
\hat{H}_{\mathrm{tot}}=\hat{H}_{\mathrm{e}}+\hat{H}_{\mu}+\hat{H}_{\mathrm{vib}}+\hat{H}_{\mathrm{rot}}
$$

where the meaning of the various terms is as follows. $\hat{H}_{\mathrm{e}}$ is the electronic Hamiltonian and is given by

$$
\hat{H}_{\mathrm{e}}=-\frac{\hbar^{2}}{2 m_{e}} \sum_{i=1}^{N_{e}} \nabla_{i}^{2}+V\left(r, \xi_{i}\right)
$$

where $V\left(r, \xi_{i}\right)$ is the Coulomb electrostatic interactions between all particles (electrons and nuclei) and we indicated with $r$ the internuclear coordinate and collectively with $\xi_{i}$ the full set of electron coordinates; $\hat{H}_{\mu}$ is the mass-polarisation term given by

$$
\hat{H}_{\mu}=-\frac{\hbar^{2}}{2 m_{N}} \sum_{i=1}^{N_{e}} \sum_{j=1}^{N_{e}} \nabla_{i} \cdot \nabla_{j}
$$

where $m_{N}$ is the total nuclear mass; $\hat{H}_{\text {vib }}$ is the vibrational kinetic energy operator and is given by

$$
\hat{H}_{\mathrm{vib}}=-\frac{\hbar^{2}}{2 \mu} \frac{\mathrm{d}^{2}}{\mathrm{~d} r^{2}}
$$

where $\mu$ is the reduced mass of the molecule. $\hat{H}_{\text {rot }}$ is the rotational Hamiltonian and can be expressed in terms of the body-fixed rotational angular momentum (AM) operator as

$$
\hat{H}_{\text {rot }}=\frac{\hbar^{2}}{2 \mu r^{2}} \hat{\mathbf{R}}^{2} .
$$


In turn, the rotational AM can be expressed as $\hat{\mathbf{R}}=\hat{\mathbf{J}}-\hat{\mathbf{L}}-\hat{\mathbf{S}}$ where $\hat{\mathbf{J}}$ is the total AM, $\hat{\mathbf{L}}$ is the electron orbital AM and $\hat{\mathbf{S}}$ is the electron spin AM. The total AM operator $\hat{\mathbf{J}}$ acts on the Euler angles $(\theta, \phi, \chi)$ relating the laboratory-fixed and the body-fixed Cartesian frame and its expression can be found, e.g., in Ref. [53]. Introducing the ladder operators $\hat{J}_{ \pm}=\hat{J}_{x} \pm i \hat{J}_{y}, \hat{S}_{ \pm}=\hat{S}_{x} \pm i \hat{S}_{y}$ and $\hat{L}_{ \pm}=L_{x} \pm i L_{y}$ we can express the rotational Hamiltonian as

$$
\begin{aligned}
\hat{H}_{\mathrm{rot}} & =\frac{\hbar^{2}}{2 \mu r^{2}}\left[\left(\hat{J}^{2}-\hat{J}_{z}^{2}\right)+\left(\hat{L}^{2}-\hat{L}_{z}^{2}\right)+\left(\hat{S}^{2}-\hat{S}_{z}^{2}\right)+\right. \\
& \left.+\left(\hat{J}_{+} \hat{S}_{-}+\hat{J}_{-} \hat{S}_{+}\right)-\left(\hat{J}_{+} \hat{L}_{-}+\hat{J}_{-} \hat{L}_{+}\right)+\left(\hat{S}_{+} \hat{L}_{-}+\hat{S}_{-} \hat{L}_{+}\right)\right] .
\end{aligned}
$$

The approach used by Duo to solve the total rovibronic Schrödinger equation with the Hamiltonian (2) follows closely the standard coupled-surface BornOppenheimer treatment $[1,58,59]$. It is assumed that one has preliminary solved the electronic motion problem with clamped nuclei

$$
\left.\left.\hat{H}_{\mathrm{e}} \mid \text { state }, \Lambda, S, \Sigma\right\rangle=V_{\text {state }}(r) \mid \text { state }, \Lambda, S, \Sigma\right\rangle
$$

for all electronic states of interest. The electronic wave functions depend on the electron coordinates $\xi_{i}$ and parametrically on the internuclear distance $r$ and can be labelled by total spin $S=0,1 / 2,1, \ldots$, projection of $\hat{\mathbf{L}}$ along the body fixed $z$ axis $\Lambda=0, \pm 1, \pm 2$, projection of $\hat{\mathbf{S}}$ along the body fixed $z$ axis $\Sigma=0, \pm 1 / 2, \pm 1, \ldots$ and by a further label 'state' $=1,2, \ldots$ which counts over the electronic curves. For $|\Lambda| \geq 1$ the spacial part of the electronic wave functions is doubly degenerate; we choose the degenerate components $\mid$ state, $\Lambda, S, \Sigma\rangle$ so that they satisfy the following conditions [55]:

$$
\begin{aligned}
\left\langle\text { state, } \Lambda, S, \Sigma\left|\hat{L}_{z}\right| \text { state }, \Lambda, S, \Sigma\right\rangle & =\Lambda, \\
\left.\hat{\sigma}_{v}(x z) \mid \text { state }, \Lambda, S, \Sigma\right\rangle & \left.=(-1)^{s-\Lambda+S-\Sigma} \mid \text { state },-\Lambda, S,-\Sigma\right\rangle,
\end{aligned}
$$

where $\hat{\sigma}_{v}(x z)$ is the symmetry operator corresponding to a reflection through the body-fixed $x z$-plane (parity operator) and $s=1$ for $\left|\Sigma^{-}\right\rangle$states and $s=0$ for all other states.

Once the potential energy curves $V_{\text {state }}(r)$ have been obtained, for example using an $a b$ initio quantum chemistry program, Duo solves the rotationless $(J=$ 0 ) one-dimensional Schrödinger given by Eq. (1) separately for each electronic curve $V_{\text {state }}(r)$, producing a set of vibrational eigenvalues $E_{v}$ and vibrational wave functions $\mid$ state, $v\rangle$, where $v=0,1, \ldots$ is the vibrational quantum number assigned on the basis of the energy ordering; technical details on this step are given in Section 2.1. A subset of $N_{v}$ (state) vibrational functions are selected to form a basis set of rovibronic basis functions defined by

$$
\mid \text { state, } J, \Omega, \Lambda, S, \Sigma, v\rangle=\mid \text { state }, \Lambda, S, \Sigma\rangle \mid \text { state }, v\rangle|J, \Omega, M\rangle \text {, }
$$


where $|J, \Omega, M\rangle$ is a symmetric-top eigenfunction [53] (a function of the Euler angles) and describes the overall rotation of the molecule as a whole, $\Omega=\Lambda+\Sigma$ and $M$ is the projection of the total angular momentum along the laboratory axis $Z$. Only combinations of $\Sigma$ and $\Lambda$ which satisfy $|\Omega| \leq \min (J,|\Lambda|+S)$ are selected in the rovibronic basis set (11). The selection of vibrational basis functions to retain can be made either by specifying an energy threshold (all vibrational states below the threshold are retained) or by specifying a maximum vibrational quantum number $v_{\max }$.

The rovibrational basis set (11) is used to solve the complete rovibronic Hamiltonian given by Eq. (2); this amounts to using an expansion in Hund's case (a) functions to solve the coupled problem. In particular, the ladder operators appearing in $\hat{H}_{\text {rot }}$ couple rovibrational states belonging to different electronic states; specifically, the non-vanishing matrix elements of the angular momentum operators in the rotational Hamiltonian (7) are given by the standard rigid-rotor expressions [60]:

$$
\begin{aligned}
\left\langle J, \Omega\left|\hat{J}_{z}\right| J, \Omega\right\rangle & =\Omega, \\
\left\langle J, \Omega\left|\hat{J}^{2}\right| J, \Omega\right\rangle & =J(J+1), \\
\left\langle J, \Omega \mp 1\left|\hat{J}_{ \pm}\right| J, \Omega\right\rangle & =\sqrt{J(J+1)-\Omega(\Omega \mp 1)},
\end{aligned}
$$

while matrix elements of the spin operators between electronic wave functions (omitting the 'state' label for simplicity) are given by

$$
\begin{aligned}
\left\langle\Lambda, S, \Sigma\left|\hat{S}_{z}\right| \Lambda, S, \Sigma\right\rangle & =\Sigma \\
\left\langle\Lambda, S, \Sigma\left|\hat{S}^{2}\right| \Lambda, S, \Sigma\right\rangle & =S(S+1) \\
\left\langle\Lambda, S, \Sigma \pm 1\left|\hat{S}_{ \pm}\right| \Lambda, S, \Sigma\right\rangle & =\sqrt{S(S+1)-\Sigma(\Sigma \pm 1)}
\end{aligned}
$$

The coupling rules for the Hamiltonian (7) are as follows; the first line in Eq. (7) is the diagonal part of the rotational Hamiltonian, i.e. is non-zero only for $\Delta S=$ $\Delta \Sigma=\Delta \Lambda=0$. The term containing $\hat{J}_{ \pm} \hat{S}_{\mp}$ is called $S$-uncoupling and is non-zero for $\Delta S=0 ; \Delta \Sigma= \pm 1 ; \Delta \Lambda=0$. The term containing $\hat{J}_{ \pm} \hat{L}_{\mp}$ is called L-uncoupling and is non-zero for $\Delta S=0 ; \Delta \Sigma=0 ; \Delta \Lambda= \pm 1$. Finally, the term containing $\hat{S}_{ \pm} \hat{L}_{\mp}$ is called spin-electronic and is non-zero for $\Delta S=0 ; \Delta \Sigma= \pm 1 ; \Delta \Lambda=\mp 1$.

Matrix elements of the orbital AM operators $\hat{L}_{x}^{2}+\hat{L}_{y}^{2} \equiv \hat{L}^{2}-\hat{L}_{z}^{2}$ and $\hat{L}_{ \pm}$when averaged over the electronic wave functions give rise to $r$-dependent curves; these can be computed by ab initio methods [61] or estimated semi-empirically, for example using quantum defect theory $[62,63]$.

The expectation value of the sum of the vibrational and the mass-polarisation Hamiltonian using the electronic wave functions gives rise $[58,64]$ to the so-called 
Born-Oppenheimer diagonal correction (also called adiabatic correction), which can be added to the Born-Oppenheimer PEC $V_{\text {state }}(r)$ if desired.

At this stage Duo builds the full Hamiltonian matrix in the basis of Eq. (11) and using the Hamiltonian operator (2), possibly complemented by supplementary terms such as spin-orbit coupling (see section 2.6 for a list of possible additional terms to the Hamiltonian). The vibrational matrix elements

$$
\left\langle\text { state }_{\lambda}, v_{\lambda}|\hat{F}(r)| \text { state }_{\mu}, v_{\mu}\right\rangle
$$

for all operators $\hat{F}(r)$ including couplings, dipole moments, corrections etc. between different vibrational basis set functions are computed and stored; note that in the equation above state ${ }_{\lambda}$ and state ${ }_{\mu}$ indicate different electronic states if $\lambda \neq \mu$.

At this point a basis set transformation is carried out, from the basis given by Eq. (11) to a symmetrized one in which the basis functions have well-defined parity; parity (even or odd) is defined with respect to inversion of all laboratoryfixed coordinates $[55,56,65,66]$ and is equivalent to the reflection operation through the molecule-fixed $x z$ plane, $\hat{\sigma}_{v}(x z)$. The parity properties of the basis functions of Eq. (11) are given by Kato [55]

$$
\left.\left.\hat{\sigma}_{v}(x z) \mid \text { state }, J, \Omega, \Lambda, S, \Sigma, v\right\rangle=(-1)^{s-\Lambda+S-\Sigma+J-\Omega} \mid \text { state }, J,-\Omega,-\Lambda, S,-\Sigma, v\right\rangle \text {, }
$$

where $s=1$ for $\left|\Sigma^{-}\right\rangle$states and $s=0$ for all other states. The symmetrized basis functions are symmetric (+) or antisymmetric (-) with respect to $\hat{\sigma}_{v}(x z)$. Use of the symmetrized basis set leads to two separate Hamiltonian blocks with defined parities.

The two parity blocks are then diagonalized (see Section 2.8 for technical details), to obtain the final rovibronic eigenvalues $E_{\lambda}^{J, \tau}$ and corresponding eigenfunctions $\phi_{\lambda}^{J, \tau}$, where $\tau= \pm 1$ is the parity quantum number, $\lambda=1,2, \ldots$ is a simple counting index. The corresponding rovibronic wave function $\phi_{\lambda}^{J, \tau}$ can be written as an expansion in the basis set (11)

$$
\phi_{\lambda}^{J, \tau}=\sum_{n} C_{\lambda, n}^{J, \tau}|n\rangle
$$

where the $C_{\lambda, n}^{J, \tau}$ are expansion coefficients and $n$ here is a shorthand index for the basis set labels 'state', $J, \Omega, \Lambda, S, \Sigma$, and $v$ :

$$
|n\rangle=\mid \text { state }, J, \Omega, \Lambda, S, \Sigma, v\rangle \text {. }
$$

As the notation above indicates, in the general case the only good quantum numbers (i.e. labels associated with the eigenvalues of symmetry operators) are the total angular momentum value $J$ and the parity $\tau$. Nevertheless, Duo analyzes the eigenvectors and assigns energy levels with the approximate quantum 
Table 1: Sample Duo energy output for AlO [47]. The energy is given in $\mathrm{cm}^{-1}$, and the exact (J, n, parity) and approximate (state, $\mathrm{v}(v)$, lambda $(\Lambda)$, spin $(S)$, sigma $(\Sigma)$, and omega $(\Omega)$ ) quantum numbers. The final column contains labels of the electronic states as given by the user and the separator || is to facilitate selecting the energy entries in the program output.

\begin{tabular}{|c|c|c|c|c|c|c|c|c|c|c|}
\hline $\mathrm{J}$ & $\mathrm{N}$ & Energy/cm & State & $\mathrm{V}$ & lambda & spin & sigma & omega & \multicolumn{2}{|c|}{ parity } \\
\hline 0.5 & 1 & 0.000000 & 1 & 0 & 0 & 0.5 & 0.5 & 0.5 & + & | |X2SIGMA+ \\
\hline 0.5 & 2 & 965.435497 & 1 & 1 & 0 & 0.5 & 0.5 & 0.5 & + & | |X2SIGMA+ \\
\hline 0.5 & 3 & 1916.845371 & 1 & 2 & 0 & 0.5 & 0.5 & 0.5 & + & | |X2SIGMA+ \\
\hline 0.5 & 4 & 2854.206196 & 1 & 3 & 0 & 0.5 & 0.5 & 0.5 & + & | |X2SIGMA+ \\
\hline 0.5 & 5 & 3777.503929 & 1 & 4 & 0 & 0.5 & 0.5 & 0.5 & + & | |X2SIGMA+ \\
\hline 0.5 & 6 & 4686.660386 & 1 & 5 & 0 & 0.5 & 0.5 & 0.5 & + & | |X2SIGMA+ \\
\hline 0.5 & 7 & 5346.116382 & 2 & 0 & 1 & 0.5 & -0.5 & 0.5 & + & | |A2PI \\
\hline 0.5 & 8 & 5581.906844 & 1 & 6 & 0 & 0.5 & 0.5 & 0.5 & + & | |X2SIGMA+ \\
\hline 0.5 & 9 & 6066.934830 & 2 & 1 & 1 & 0.5 & -0.5 & 0.5 & + & | |A2PI \\
\hline 0.5 & 10 & 6463.039443 & 1 & 7 & 0 & 0.5 & 0.5 & 0.5 & + & | |X2SIGMA+ \\
\hline 0.5 & 11 & 6778.997803 & 2 & 2 & 1 & 0.5 & -0.5 & 0.5 & + & | |A2PI \\
\hline 0.5 & 12 & 7329.427637 & 1 & 8 & 0 & 0.5 & 0.5 & 0.5 & + & | |X2SIGMA+ \\
\hline 0.5 & 13 & 7483.145675 & 2 & 3 & 1 & 0.5 & -0.5 & 0.5 & + & | |A2PI \\
\hline 0.5 & 14 & 8159.170405 & 2 & 4 & 1 & 0.5 & -0.5 & 0.5 & + & | |A2PI \\
\hline 0.5 & 15 & 8201.467744 & 1 & 9 & 0 & 0.5 & 0.5 & 0.5 & + & | |X2SIGMA+ \\
\hline 0.5 & 16 & 8857.266385 & 2 & 5 & 1 & 0.5 & -0.5 & 0.5 & + & $\perp \mathrm{A} 2 \mathrm{PI}$ \\
\hline
\end{tabular}

numbers 'state', $v, \Lambda, \Sigma$, and $\Omega$ on the basis of the largest coefficient in the basis set expansion (21). It should be noted that the absolute signs of $\Lambda$ and $\Sigma$ are not well defined, only their relative signs are. This is related to the symmetry properties of the eigenfunctions of the Hamiltonian (2), which are 50/50 symmetric and antisymmetric mixtures of the $|\Lambda, \Sigma\rangle$ and $|-\Lambda,-\Sigma\rangle$ contributions. Therefore the absolute value of the quantum number $\Omega$ is required additionally in order to fully describe the spin-electronic-rotational contribution. In situations where some couplings are absent some approximate quantum numbers can become exact; for example, in the absence of spin-orbit and spin-spin interactions the basis functions (11) with different values of spin $S$ do not interact and, hence, $S$ becomes a "good" quantum number. As another example, without the presence of ${ }^{3} \Sigma^{-}$or ${ }^{1} \Pi$ states there is no mechanism for the ro-vibrational functions of a ${ }^{1} \Sigma^{+}$ state to interact with other electronic states and therefore the corresponding eigenfunctions will have well defined values of $S=\Sigma=\Lambda=0$.

Table 1 gives an example of a Duo output with the energy term values computed for the case of the first three electronic states, $X^{2} \Sigma^{+}, A^{2} \Pi$, and $B^{2} \Sigma^{+}$, of $\mathrm{AlO}[47]$.

\subsection{Solution of the uncoupled vibrational problem}

The main method of solving the radial equation used by Duo is the so-called sinc DVR (discrete variable representation); this method (or closely related ones) 
has been independently applied to the one-dimensional Schrödinger equation by various authors $[9,10,67,68]$.

In this method the $r$ coordinate is truncated to an interval $\left[r_{\min }, r_{\max }\right]$ and discretized in a grid of $N_{p}$ uniformly spaced points $r_{i}=r_{\min }+i \Delta r$ (where $i \in$ $\left.\left[0, N_{p}-1\right]\right)$ with grid step $\Delta r=\left(r_{\max }-r_{\min }\right) /\left(N_{\mathrm{p}}-1\right)$. The Schrödinger Eq. (1) is then transformed to an ordinary matrix eigenvalue problem

$$
(\mathbf{T}+\mathbf{V}) \vec{\psi}_{v}=E_{v} \vec{\psi}_{v}
$$

where $\mathbf{T}$ is the matrix representing the kinetic energy and is given in the sinc method by $[68,69]$

$$
T_{i j}=\frac{\hbar^{2}}{2 \mu(\Delta r)^{2}} \begin{cases}\frac{\pi^{2}}{3} & i=j \\ 2 \frac{(-1)^{i-j}}{(i-j)^{2}} & i \neq j\end{cases}
$$

and $\mathbf{V}=\operatorname{diag}\left[V\left(r_{0}\right), V\left(r_{1}\right), \ldots, V\left(r_{N_{p}-1}\right)\right]$ while the vector $\vec{\psi}_{v}$ contains the values of $\psi(r)$ at the grid points. The resulting $N_{p} \times N_{p}$ real symmetric matrix $\mathbf{H}$ is then diagonalized (see section 2.8 for details). The sinc DVR method usually provides very fast (faster than polynomial) convergence of the calculated energies and wave functions with respect to the number of the grid points, $N_{p}$. Figure $1 a$ ) shows the convergence for three $J=0$ energy levels of a Morse potential, showing a rate of convergence approximately exponential with respect to the number of grid points.

Duo obtains all integrals over vibrational coordinates by summation over the grid points:

$$
\int_{r_{\min }}^{r_{\max }} \psi_{\lambda}(r) F(r) \psi_{\mu}(r) \mathrm{d} r=\Delta r \sum_{i=0}^{N_{p}-1} \psi_{\lambda}\left(r_{i}\right) F\left(r_{i}\right) \psi_{\mu}\left(r_{i}\right) .
$$

The rectangle rule is simple and exponentially accurate for integration over infinite range of functions which decay fast (exponentially or faster) and which do not have singularities in the complex plane close to the real axis [70]. We illustrate in fig. $1 b)$ the quick convergence of matrix elements of the type $\left\langle 0\left|\left(r-r_{e}\right)^{4}\right| v\right\rangle$ for a Morse potential; analytical formulae for matrix elements of this kind are available from the literature $[71,72]$ and were used to obtain exact reference values. In plot $1 b$ ) it is apparent that the accuracy of matrix elements does not improve beyond a certain value; for example, the matrix elements $\left\langle 0\left|\left(r-r_{e}\right)^{4}\right| 10\right\rangle$ always has less than about 10 significant digits no matter how many points are used. This behaviour is completely normal and expected when performing floating-point calculations with a fixed precision; Duo uses double precision numbers with a machine epsilon $\varepsilon=2 \times 10^{-16}[73]$ and the expected relative error due to the finite precision in the 

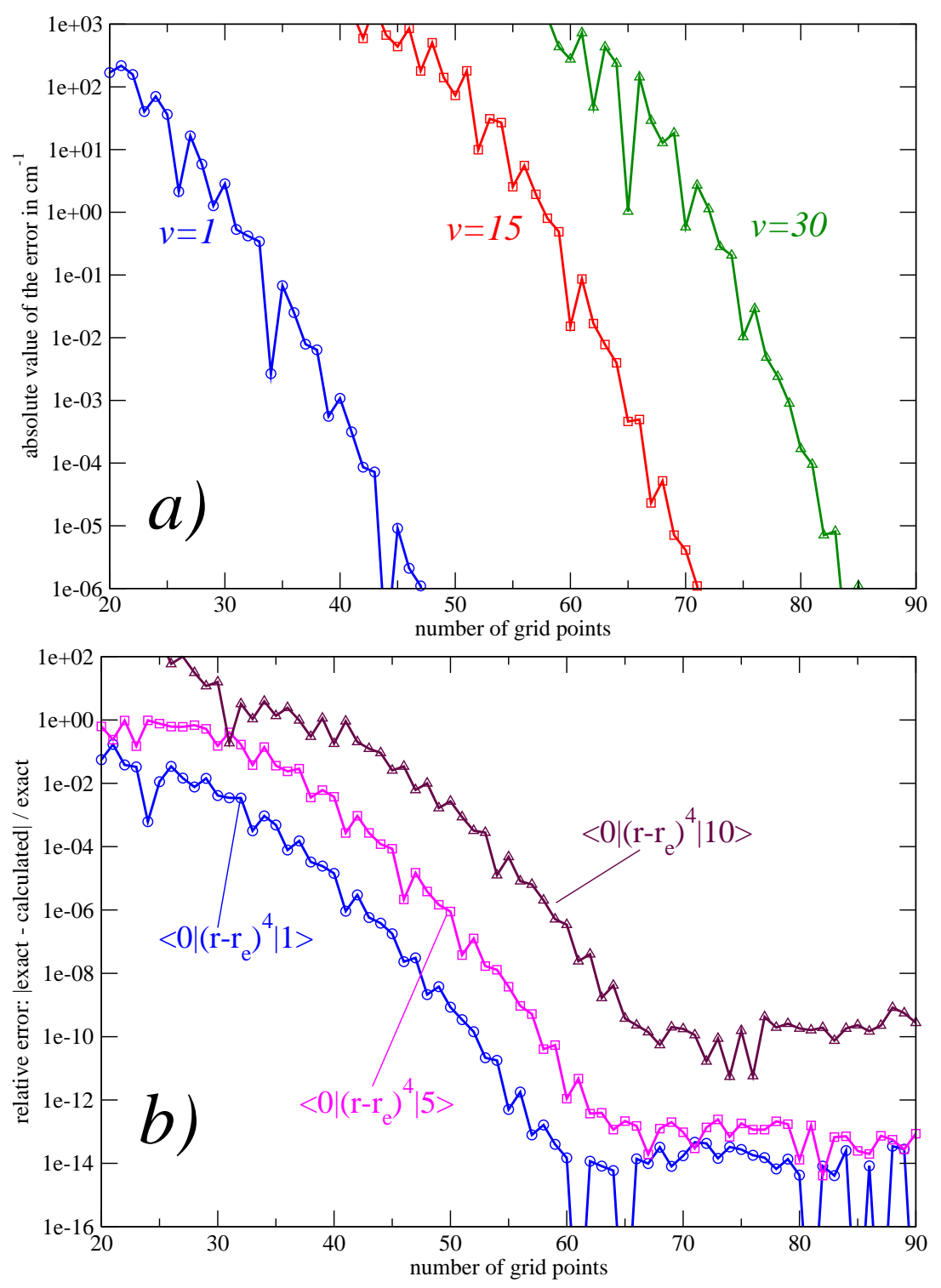

Figure 1: Illustrative examples of the fast convergence for energies (plot $a$ ) and matrix elements (plot $b$ ) using the sinc DVR method; in both cases the rate of convergence is approximately exponential with respect to the number of grid points. Results are for a Morse potential which approximately models the ground electronic state of the CO molecule, $r_{e}=1.1283 \AA, D_{e}=90674 \mathrm{~cm}^{-1}$, $\omega=2169.814 \mathrm{~cm}^{-1}$ with atomic masses for carbon and oxygen. A uniformly spaced grid was used, keeping fixed $r_{\text {min }}=0.7 \AA, r_{\max }=2.0 \AA$. In plot $a$ ) we show absolute errors for the $v=1, v=15$ and $v=30$ energy levels; in plot $b$ ) we show relative errors of matrix elements of the type $\left\langle 0\left|\left(r-r_{e}\right)^{4}\right| v\right\rangle$; the flattening of the error for large numbers of grid points is due to the numerical error present in floating point calculations (see text). 
sum given by eq. (25) is given by, indicating with $S$ the value of the sum performed with infinite accuracy and with $\hat{S}$ the value obtained with finite accuracy:

$$
\frac{|S-\hat{S}|}{S} \approx \varepsilon N_{p} \frac{\sum_{i}\left|\psi_{\lambda}\left(r_{i}\right) F\left(r_{i}\right) \psi_{\mu}\left(r_{i}\right)\right|}{\left|\left(\sum_{i} \psi_{\lambda}\left(r_{i}\right) F\left(r_{i}\right) \psi_{\mu}\left(r_{i}\right)\right)\right|}
$$

The expression above implies that whenever the matrix element of a function $F$ comes out very small with respect to the value of $|F|$ significant digits will be lost; there are techniques such as Kahan compensated summation [73] which reduce the error above by a factor $N_{p}$ but these have not been implemented at this time.

A prime example of this situation if given by the line intensities of very high vibrational overtones; in a recent study Medvedev et al. [74] observed that matrix elements of the type $|\langle 0|\mu(r)| v\rangle|$ for the CO molecule when computed with double precision floating-point arithmetic decrease approximately exponentially (as expected on the basis of theoretical models and as confirmed by quadruple precision calculations) for $v \lesssim 25$, when they reach the value of about $10^{-15} \mathrm{D}$. This situation is fully expected on the basis of the considerations above but it should never constitute a problem in practice.

Apart from the sinc DVR, Duo implements finite-difference (FD) methods for solving the uncoupled vibrational problem, where the kinetic energy operator $\mathbf{T}$ in Eq. (23) can be approximated using, for example, a 5-point central FD5 formulae:

$$
T_{i j}=\frac{\hbar^{2}}{2 \mu(\Delta r)^{2}} \begin{cases}5 / 2 & i=j, \\ -4 / 3 & |i-j|=1 \\ 1 / 12 & |i-j|=2\end{cases}
$$

and furthermore with $T_{11}=T_{N_{p} N_{p}}=29 / 12$. Note that the expression above gives incorrect results for the first two and last two grid points, but this does not matter as long as the grid truncation parameters $r_{\min }$ and $r_{\max }$ are chosen so that $\psi_{v} \approx 0$ near the borders of the grid.

The formulae (27) lead to a symmetric pentadiagonal banded matrix, which can in principle be diagonalized more efficiently than a generic dense matrix. However, the convergence of the eigenvalues $E_{v}$ is much slower, with error decreasing as $(\Delta r)^{4}$ instead of $e^{-\alpha /(\Delta r)}$.

\subsection{Levels lying close to dissociation}

A general requirement for convergence is that both the inner and the outer grid truncation values $r_{\min }$ and $r_{\max }$ should be chosen such that $V_{\text {state }}\left(r_{\min }\right)$ and $V_{\text {state }}\left(r_{\max }\right)$ are both much larger than $E_{v}$. A problem arises when one is trying to converge states very close to the dissociation limit, as such loosely bound states can extend to very large values of $r$ and therefore require an excessive number of points when a uniformly spaced grid is used; this is illustrated in fig. 2. 


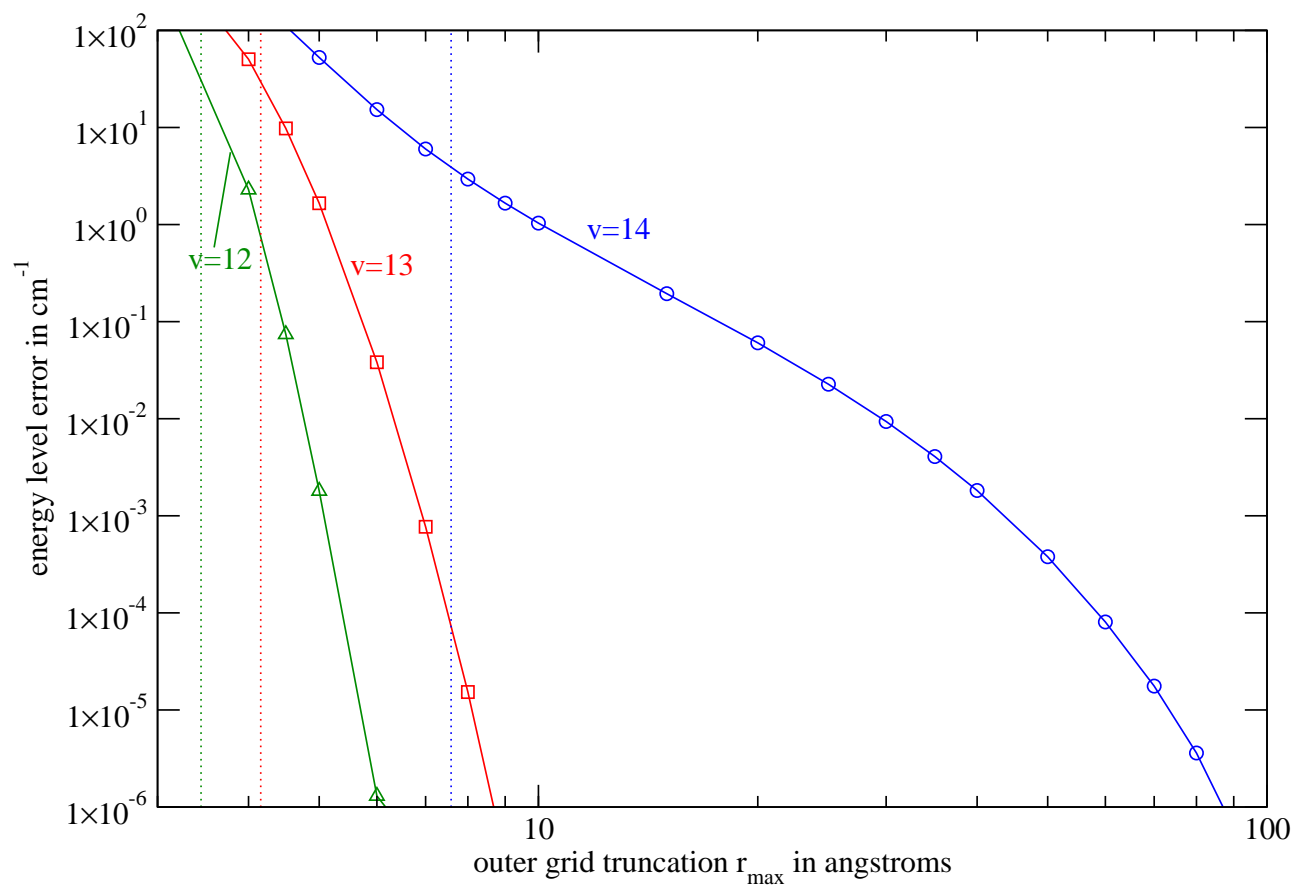

Figure 2: Illustrative example of the effect of the outer grid truncation parameter $r_{\max }$ on energy levels close to dissociation. Data are relative to a Morse potential with $D_{e}=12728.237432 \mathrm{~cm}^{-1}, \omega_{e}=1751.124844 \mathrm{~cm}^{-1}, r_{e}=1 \AA, \mu=1 \mathrm{Da}$. This potential supports 15 bound states $(v=0$ to $v=14)$ and we consider in this example the three highest-energy ones, with energies $E_{12}=-250.7130 \mathrm{~cm}^{-1}$, $E_{13}=-65.2068 \mathrm{~cm}^{-1}, E_{14}=-0.1000 \mathrm{~cm}^{-1}$. In all calculation we fixed $r_{\min }=$ $0.1 \AA$ and the grid step $\Delta r$ to $0.05 \AA$. The dotted vertical lines are the outer turning points for the three states, i.e. the points $r_{\text {out }}$ such that $V\left(r_{\text {out }}\right)=E_{v}$; the error in the computed energy levels is expected to decrease exponentially when $r_{\max }>$ $r_{\text {out }}$. The plot shows that to converge the last energy level $E_{14}$ a very large $r_{\max }$ is required, which in turn leads to a large number of grid points when they are uniformly spaced. Specifically, to converge $E_{12}$ to $10^{-6} \mathrm{~cm}^{-1}$ it is sufficient to choose $r_{\max }>6 \AA$, leading to 120 points; for $E_{13}$ we need $r_{\max }>9 \AA$ and 180 points; for $E_{14}$ we need $r_{\max }>90 \AA$ and 1500 points. 
Excited states of alkali diatoms such as $\mathrm{Li}_{2}$ [75], $\mathrm{Na}_{2}$ [76] or $\mathrm{K}_{2}$ [77] constitute an important class of systems for which large $r_{\max }$ are needed; such systems are prime choices for studies of ultracold atoms and molecules [78] and often require grids extending up to several tens [75, 76] or even hundreds [77] of Angstroms.

In such cases it may be beneficial to use a non uniform grid; Duo implements the adaptive analytical mapping approach of Meshkov et al [79] and offers several mapping choices, which are described in the manual. However, at this time support for non uniform grids should be considered experimental and they cannot be used in combination with the sinc DVR method but only with the less efficient 5point finite-difference one. Indicatively we recommend considering non-uniform grids only when $r_{\text {max }}$ is required to be larger than $\approx 50 \AA$.

\subsection{States beyond the dissociation limit}

Potential curves with local maxima higher than the dissociation limit of the potential for $r \rightarrow+\infty$ may support shape resonances, i.e. metastable states in which the two atoms are trapped for a finite time in the potential well but eventually dissociate. Such states are also known as quasibound or tunnelling predissociation states. For $J>0$ the rotational potential will practically always introduce such a maximum, and the corresponding quasibound levels are known as orbiting resonances or rotationally predissociating states, see fig. $3 a$ ) for an example.

Several techniques have been developed to deal with quasibound states, most notably in the context of diatomic molecules by Le Roy and co-workers [80-88]. At the moment Duo does not provide any explicit functionality to treat quasibound states, although we plan to rectify this deficiency in future versions.

Nevertheless, long-lived quasibound states (i.e., narrow resonance) can be identified using the present version of Duo by using the so-called stabilization method [89-94]. In one version of this approach energy levels are computed for increasing values of the outer grid truncation $r_{\max }$ and then plotted as function of $r_{\max }$; quasibound states manifests themselves by being relatively stable with respect to increase of $r_{\max }$ and undergo a series of avoided crossings, see fig. $3 \mathrm{~b}$ ) for an example. From an analysis of these curves it is also possible to compute the lifetime of the quasibound state [93].

\subsection{Printing the wave functions}

Both the $J=0$ vibrational basis functions $\mid$ state, $v\rangle$, see eq. (11), and the final $\left(J>0\right.$, electronically coupled or both) rovibronic wave functions coefficients $C_{\lambda, n}^{J, \tau}$, see eq. (21), can be written to a file for further analysis, e.g. for plotting purposes or for the computation of $g$ factors; see the manual for details. 

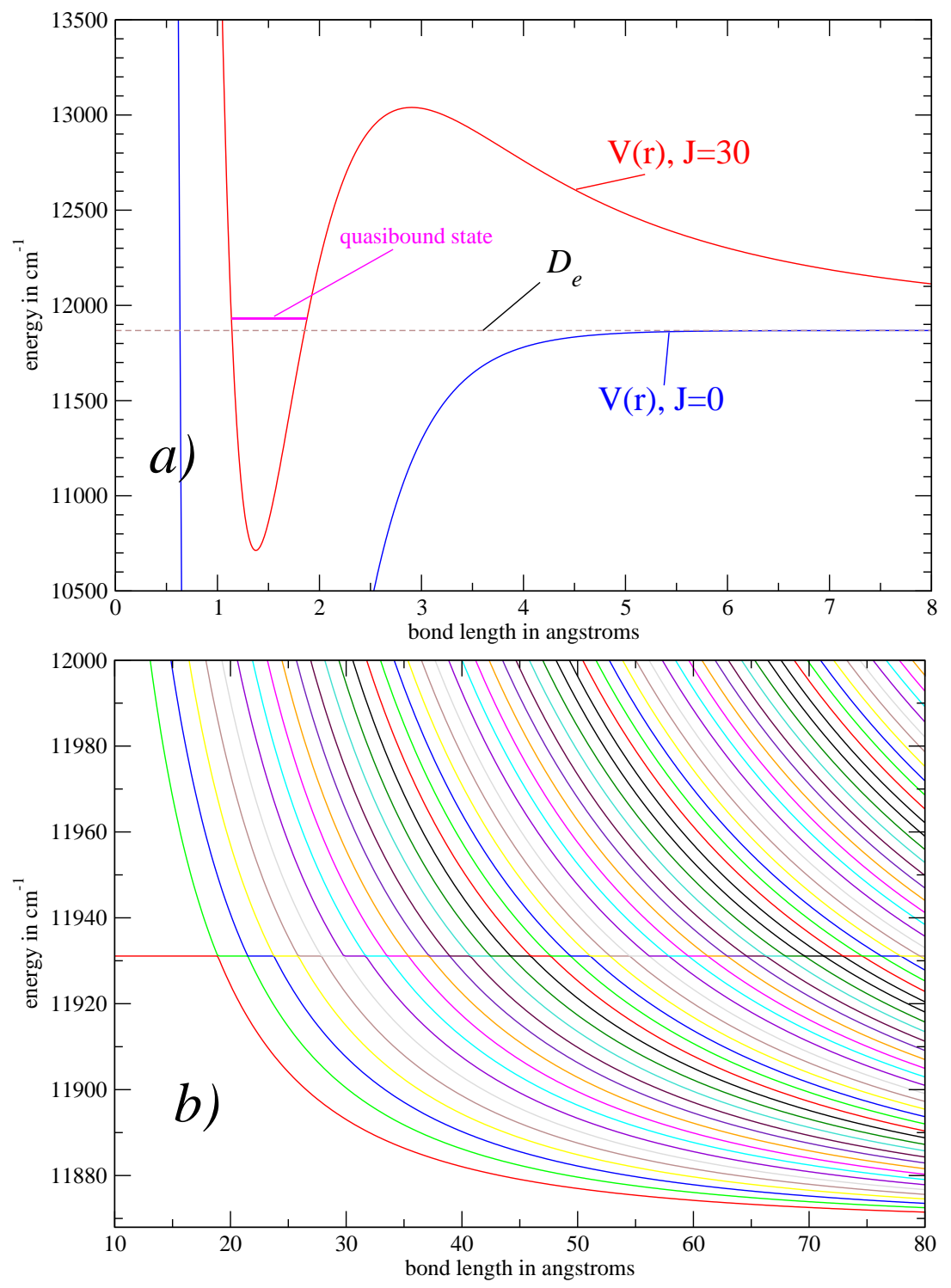

Figure 3: Example of a quasibound, orbiting resonance state. Plot $a$ ): Morse potential for $J=0$ and $J=30$ (same parameters as in fig. 2); the potential for $J=30$ has a local maximum higher than the dissociation limit $D_{e}$ and supports one quasibound state with energy $E=11931.1 \mathrm{~cm}^{-1}$. Plot $b$ ): eigenvalues for $E>D_{e}$ as a function of the outer grid truncation $r_{\max }$. The quasibound state manifests itself as a series of avoided crossings. 


\subsection{Convergence of rotationally excited states}

In our approach $J>0$ calculations are performed using a basis expansion in terms of the $J=0$ wave functions. As a guideline it was found by numerical experimentation that in order to obtain converged results for rotationally excited states up to $v_{\max }$ one has to use a vibrational $J=0$ basis set of size only slightly larger than $v_{\max }$ and that a reasonable minimum value for the size of the vibrational basis set is given by $1.25 \times v_{\max }+2$. For example, to converge rotationally excited levels up to $v=30$ it should be sufficient to use a vibrational basis set of size 40 .

\subsection{Additional terms in the Hamiltonian}

Duo supports the inclusion of a number of terms additional to the non-relativistic Hamiltonian (2) caused by spin-orbit $\hat{H}_{\mathrm{SO}}$, spin-rotational $\hat{H}_{\mathrm{SR}}$, spin-spin $\hat{H}_{\mathrm{SS}}$ and $\Lambda$-doubling $\hat{H}_{\mathrm{LD}}$ interactions [55, 95-99]:

1. The Breit-Pauli spin-orbit operator $\hat{H}_{\text {SO }}$ [99-102] has non-zero matrix elements between electronic states obeying the following coupling rules [95]: $\Delta S=0, \pm 1 ; \Delta \Lambda=0, \pm 1 ; \Delta \Omega=0$; if $\Delta S=0$ and $\Sigma^{\prime}=\Sigma^{\prime \prime}=0$ the matrix elements is zero (this last rule implies that singlet-to-singlet matrix elements are zero); $\Sigma^{+}$electronic states may have non-zero matrix elements with $\Sigma^{-}$ states but $\Sigma^{ \pm} \leftrightarrow \Sigma^{ \pm}$matrix elements are zero; finally, in case of homonuclear diatomics, only $g \leftrightarrow g$ and $u \leftrightarrow u$ matrix elements are non-zero.

The diagonal SO matrix elements $\left\langle\Lambda, S, \Sigma\left|\hat{H}_{\mathrm{SO}}\right| \Lambda, S, \Sigma\right\rangle$ determine the spinorbit splitting of a multiplet ${ }^{2 S+1} \Lambda$, where $S>0$ and $\Lambda>0$. Both diagonal and off-diagonal matrix elements of the spin-orbit Hamiltonian can be obtained as functions of $r$ using quantum chemistry programs.

2. The nonzero diagonal and off-diagonal matrix elements of $\hat{H}_{\mathrm{SR}}$ operator are given by

$$
\begin{aligned}
\left\langle\Lambda, S, \Sigma\left|\hat{H}_{\mathrm{SR}}\right| \Lambda, S, \Sigma\right\rangle & =\frac{\hbar^{2}}{2 \mu r^{2}} \gamma^{\mathrm{SR}}(r)\left[\Sigma^{2}-S(S+1)\right] \\
\left\langle\Lambda, S, \Sigma\left|\hat{H}_{\mathrm{SR}}\right| \Lambda, S, \Sigma \pm 1\right\rangle & =\frac{\hbar^{2}}{4 \mu r^{2}} \gamma^{\mathrm{SR}}(r)[S(S+1)-\Sigma(\Sigma \pm 1)]^{1 / 2} \\
& \times[J(J+1)-\Omega(\Omega \pm 1)]^{1 / 2}
\end{aligned}
$$

where $\gamma^{\mathrm{SR}}(r)$ is a dimensionless function of $r$.

3. The diagonal matrix elements of the $\hat{H}_{\mathrm{SS}}$ operator are taken in the phenomenological form

$$
\left\langle\Lambda, S, \Sigma\left|\hat{H}_{\mathrm{SS}}\right| \Lambda, S, \Sigma\right\rangle=\frac{2}{3} \lambda^{\mathrm{SS}}(r)\left[3 \Sigma^{2}-S(S+1)\right]
$$

Both $\gamma^{\mathrm{SR}}(r)$ and $\lambda^{\mathrm{SS}}(r)$ functions can be obtained either $a b$ initio or empirically. 
4. The lambda-doubling (LD) couplings for $\mathrm{a}^{2 S+1} \Pi$ state in the $\Lambda$-representation (Eq. (11)) are of the following three types [98]:

$$
\hat{H}_{\mathrm{LD}}=\frac{1}{2} \alpha_{o p q}^{L D}(r)\left(\hat{S}_{+}^{2}+\hat{S}_{-}^{2}\right)-\frac{1}{2} \alpha_{p 2 q}^{\mathrm{LD}}(r)\left(\hat{J}_{+} \hat{S}_{+}+\hat{J}_{-} \hat{S}_{-}\right)+\frac{1}{2} q^{\mathrm{LD}}(r)\left(\hat{J}_{+}^{2}+\hat{J}_{-}^{2}\right),
$$

where $\alpha_{o p q}^{\mathrm{LD}}$ and $\alpha_{p 2 q}^{\mathrm{LD}}$ are related to the conventional terms as given by Brown and Merer [98]:

$$
\begin{aligned}
& \alpha_{o p q}^{\mathrm{LD}}=o^{\mathrm{LD}}+p^{\mathrm{LD}}+q^{\mathrm{LD}} \\
& \alpha_{p 2 q}^{\mathrm{LD}}=p^{\mathrm{LD}}+2 q^{\mathrm{LD}} .
\end{aligned}
$$

5. It is now well-established that, at least for ${ }^{1} \Sigma$ states, the small shifts to energy levels due to non-adiabatic interactions with remote states (as opposed to near-degenerate ones) can be accurately modelled by modifying the vibrational and rotational energy operators in the Hamiltonian [64, 103-107]; specifically, the vibrational energy operator in Eq. (5) is replaced by

$$
-\frac{\hbar^{2}}{2 \mu} \frac{\mathrm{d}}{\mathrm{d} r}(1+\beta(r)) \frac{\mathrm{d}}{\mathrm{d} r}
$$

while the rotational kinetic energy operator $\hbar^{2} / 2 \mu r^{2}$ in Eq. (7) should be replaced by

$$
\frac{\hbar^{2}}{2 \mu r^{2}}(1+\alpha(r)) .
$$

The functions $\alpha(r)$ and $\beta(r)$ are sometimes referred to as Born-Oppenheimer breakdown (BOB) curves [108] and can also be interpreted as introducing position-dependent vibrational and rotational masses; they are sometimes expressed in terms of the dimensionless $g$-factor functions $g_{\mathrm{v}}$ and $g_{\mathrm{r}}$ by $\alpha(r)=\left(m_{e} / m_{p}\right) g_{\mathrm{r}}(r)$ and $\beta(r)=\left(m_{e} / m_{p}\right) g_{\mathrm{v}}(r)$. The rotational $g_{\mathrm{r}}$ function can be determined experimentally by analysis of the Zeeman splitting of energy levels due to an external magnetic field [109].

\subsection{Representation of the couplings}

Duo assumes that the coupling matrix elements and the transition dipole moments are given in the representation of the basis functions (11) corresponding to Hund's case (a). In this representation the $\hat{L}_{z}$ component is diagonal and has a signed value $\Lambda$ (see Eq.(13)) and therefore it will be referred to as the $\Lambda$ representation. It can be shown that by choosing appropriate phase factors for the electronic wave functions $\mid$ state, $\Lambda, S, \Sigma\rangle$ all coupling matrix elements in the $\Lambda$-representation can be made real; note that in this representation the electronic wave functions are complex numbers, as they contain a factor of the kind $e^{i \Lambda \phi}$, 
where $\phi$ is the angle corresponding to rotation around the $z$ axis [65]. On the other hand quantum chemistry programs such as MoLPRo [110] normally work with real wave functions and consequently compute matrix elements in this representation, which we call Cartesian as the electronic wave functions are ultimately expressed in terms of atom-centred Cartesian components [100] $|x\rangle,|y\rangle,|z\rangle,|x y\rangle$ etc.

Duo can accept input in either the Cartesian or the $\Lambda$-representation. For the Cartesian-representation Duo will then transform these inputs to the $\Lambda$-representation as follows:

$$
| \pm \Lambda\rangle=\left[C_{1}^{ \pm|\Lambda|}|1\rangle+C_{2}^{ \pm|\Lambda|}|2\rangle\right]
$$

where 1 and 2 denote Cartesian components $x, y, z, x y, \ldots$ that correspond to the $A_{1}, B_{1}$ and $A_{2}, B_{2}$ Abelian point group symmetries, respectively. $C_{\alpha}^{\Lambda}$ are the elements of the unitary transformation from the Cartesian to the $\Lambda$-representation. The obvious way to reconstruct this transformation is to diagonalize the Cartesian representation of the $\hat{L}_{z}$ matrix. Thus the transformed matrix elements in the $\Lambda$-representation are given by

$$
\left\langle\Lambda \Sigma|\hat{A}| \Lambda^{\prime} \Sigma^{\prime}\right\rangle=\sum_{\alpha, \beta=0,1,2}\left(C_{\alpha}^{\Lambda}\right)^{*} C_{\beta}^{\Lambda^{\prime}}\left\langle\alpha \Sigma|\hat{A}| \beta \Sigma^{\prime}\right\rangle
$$

or, in tensorial form $\hat{A}^{\text {Duo }}=\hat{C}^{\dagger} \hat{A} \hat{C}$ where $\alpha=\beta=0$ correspond to a ${ }^{2 S+1} \Sigma(\Lambda=0)$ electronic state with $C_{0}^{0}=1$.

In principle all Cartesian matrix elements $\left\langle\alpha \Sigma|\hat{A}| \beta \Sigma^{\prime}\right\rangle$ must be provided to perform the transformation in Eq. (36). However, by means of the coupling rules all non-zero matrix elements $\left\langle\alpha \Sigma|\hat{A}| \beta \Sigma^{\prime}\right\rangle$ can be related to only one, non-zero reference matrix element. For example, the matrix element $\left\langle\Lambda=\Sigma=0\left|H^{\mathrm{SO}}\right| \Lambda^{\prime}=\Sigma^{\prime}=\right.$ $\pm 1\rangle$ between ${ }^{1} \Sigma$ and ${ }^{3} \Pi$ is zero because it corresponds to a simultaneous change of $\Lambda$ and $\Sigma$ by \pm 1 . This property together with the help of Eq. (35) allows one to use the non-zero spin-orbit matrix elements $\left\langle 0, \Sigma=0\left|H^{\mathrm{SO}}\right| 2, \Sigma=1\right\rangle$ as a reference and to reconstruct all other non-zero Cartesian component by

$$
\left\langle 0, \Sigma=0\left|H^{\mathrm{SO}}\right| 1, \Sigma=1\right\rangle=-\frac{C_{2}}{C_{1}}\left\langle 0, \Sigma=0\left|H^{\mathrm{SO}}\right| 2, \Sigma=1\right\rangle,
$$

as required for Eq. (36).

Off-diagonal matrix elements of the various operators included into our model, i.e. the various couplings between electronic states, are subject in actual calculations to arbitrary changes of sign due to the sign indeterminacy of the electronic wave functions computed at different geometries. Often the phases of each $a b$ initio coupling $F(r)$ have to be post-processed in order to provide a consistent, smooth function of $r$. It is important that the relative phases between different elements preserved. This issue is illustrated graphically by Patrascu et al. [47], 
where different $a b$ initio coupling curves of AlO obtained with MoLPRo were presented. Transition dipole moment functions, discussed in the next section, also may exhibit phase changes [111], which should be corrected using the same phase convention used for other matrix elements [47].

\subsection{Computational considerations}

Duo uses the matrix diagonalization routines DSYEV or, optionally, DSYEVR from the LAPACK library [112]. The subroutine DSYEVR uses the multiple relatively robust representations algorithm and is expected to be faster than DSYEV, which is based on the QR algorithm [113, 114]; however, the current version of DSYEVR is poorly parallelized and therefore not recommended for parallel environments.

The dimension of the final rovibrational Hamiltonian matrix depends on the number of vibrational functions selected, the number of electronic states present, the spin multiplicities of the electronic states and the $J$ quantum number. For example, for $N$ electronic states, $N_{v}$ vibrational functions are retained for each of them and denoting with $\bar{m}$ the average spin multiplicity, the size of the Hamiltonian matrix is approximately given by $N \times N_{v} \times \bar{m}$ and the size of the $\tau= \pm$ parity matrix to be diagonalized is half of this value. The size of each block of the Hamiltonian reaches dimensions of the order of a thousand only for rather complicated cases (e.g., $N=10, N_{v}=40$ and $\bar{m}=5$ ) and consequently the time taken to compute the energy levels for a given $J$ is usually only a small fraction of a second.

\section{Line intensities and line lists}

The Einstein coefficient $A_{f i}$ (in $1 / s$ ) for a transition $\lambda_{f} \leftarrow \lambda_{i}$ is computed as

$$
\begin{aligned}
A_{f i}= & \frac{64 \times 10^{-36} \pi^{4}}{3 h}\left(2 J_{i}+1\right) \tilde{v}^{3} \sum_{t=-1,0,1} \mid \sum_{n_{i}, n_{f}}\left(C_{\lambda_{f}, n_{f}}^{J_{f}, \tau_{f}}\right)^{*} C_{\lambda_{i}, n_{i}}^{J_{i}, \tau_{i}} \\
& \left.(-1)^{\Omega_{i}}\left(\begin{array}{ccc}
J_{i} & 1 & J_{f} \\
\Omega_{i} & t & -\Omega_{f}
\end{array}\right)\left\langle v_{f}\left|\bar{\mu}_{t}^{f, i}(r)\right| v_{i}\right\rangle\right|^{2},
\end{aligned}
$$

where $\bar{\mu}_{t}(t=-1,0,1)$ are the electronically averaged body-fixed components of the electric dipole moment (in Debye) in the irreducible representation

$$
\bar{\mu}_{0}=\bar{\mu}_{z} ; \quad \bar{\mu}_{ \pm 1}=\mp \frac{1}{\sqrt{2}}\left(\bar{\mu}_{x} \pm i \bar{\mu}_{y}\right),
$$

and the index $n$ is defined by Eq. (22). The vibrationally averaged transition dipole moments $\left\langle v_{f}\left|\bar{\mu}_{t}^{f, i}(r)\right| v_{i}\right\rangle$ are computed using the vibrational wave functions $|v\rangle \equiv$ $\psi_{v}(r)$. 
The absorption line intensity is then given by

$$
I(f \leftarrow i)=\frac{g_{\mathrm{ns}}\left(2 J_{f}+1\right) A_{f i}}{8 \pi c \tilde{v}^{2}} \frac{e^{-c_{2} \tilde{E}_{i} / T}\left(1-e^{-c_{2} \tilde{v}_{i f} / T}\right)}{Q(T)},
$$

where $Q(T)$ is the partition function defined as

$$
Q(T)=g_{\mathrm{ns}} \sum_{i}\left(2 J_{i}+1\right) e^{-c_{2} \tilde{E}_{i} / T}
$$

$g_{\mathrm{ns}}$ is the nuclear statistical weight factor, $c_{2}=h c / k_{B}$ is the second radiation constant, $\tilde{E}_{i}=E_{i} / h c$ is the term value, and $T$ is the temperature in K. For heteronuclear molecules $g_{\mathrm{ns}}$ is a total number of combinations of nuclear spins as given by $g_{\mathrm{ns}}=\left(2 I_{b}+1\right)\left(2 I_{a}+1\right)$, where $I_{a}$ and $I_{b}$ are the corresponding nuclear spins. For a homonuclear molecules, these combinations are distributed among the four symmetries $+s,-s,+a,-a$, where $+/-$ is the parity of the molecule with respect to $\sigma_{v}$ and $s / a$ is the property of the total rovibronic wave function to be symmetric/asymmetric upon upon inversion [55]. In the representation of $C_{2 v}$ point group symmetry, this corresponds to $A_{1}, A_{2}, B_{1}$, and $B_{2}$. Thus, for the case $I \equiv I_{a}=I_{b}$ two different values $g_{\text {ns }}$ are necessary and these depend on whether the nuclei are fermions ( $I$ is half-integer) or bosons ( $I$ integer) $[115,116]$ :

$$
g_{\mathrm{ns}}= \begin{cases}\frac{1}{2}\left[(2 I+1)^{2}-(2 I+1)\right], & \text { Fermi, } s \text { and Bose, } a \\ \frac{1}{2}\left[(2 I+1)^{2}+(2 I+1)\right], & \text { Fermi, } a \text { and Bose, } s .\end{cases}
$$

For example, carbon ${ }^{12} \mathrm{C}$ has $I=0$ and therefore for the $\mathrm{C}_{2}$ molecule $g_{\mathrm{ns}}$ are 1 for $A_{1}, A_{2}$ and 0 for $B_{1}, B_{2}$ states, respectively.

The computed Einstein $A$ coefficients can be used to compute radiative lifetimes of individual states and cooling functions in a straightforward manner [117].

\subsection{Line list format}

A line list is defined as a catalogue of transition frequencies and intensities [118]. In the basic ExoMol format [118], adopted by Duo, a line list consists of two files: 'States' and 'Transitions'; an example for the molecule AlO is given in Tables 2 and 3. The 'States' file contains energy term values supplemented by the running number $n$, total degeneracy $g_{n}$, rotational quantum number $J_{n}$ (all obligatory fields) as well as quantum numbers $v, \Lambda$, parity $( \pm), \Sigma, \Omega$ and the electronic state label (e.g. X2Sigma+). The 'Transitions' file contains three obligatory columns, the upper and lower state indexes $n_{f}$ and $n_{i}$ which are running numbers from the 'State' file, and the Einstein coefficient $A_{f i}$. For the convenience we also provide the wavenumbers $\tilde{v}_{i f}$ as the column 4. The line list in the ExoMol format can be used to simulate absorption or emission spectra for any temperature in a general way. Note that ExoMol format has recently been significantly extended [119] but structure of the States and Transitions file has been retained. 
Table 2: Extract from the output 'State' file produced by Duo for the ${ }^{27} \mathrm{Al}{ }^{16} \mathrm{O}$ molecule [48].

\begin{tabular}{rrrrrrlrrrr}
\hline$n$ & \multicolumn{1}{c}{$\tilde{E}$} & $g$ & $J$ & $+/-$ & $e / f$ & State & $v$ & $|\Lambda|$ & $|\Sigma|$ & $|\Omega|$ \\
\hline 1 & 0.000000 & 12 & 0.5 & + & $\mathrm{e}$ & X2SIGMA & 0 & 0 & 0.5 & 0.5 \\
2 & 965.435497 & 12 & 0.5 & + & $\mathrm{e}$ & X2SIGMA & 1 & 0 & 0.5 & 0.5 \\
3 & 1916.845371 & 12 & 0.5 & + & $\mathrm{e}$ & X2SIGMA & 2 & 0 & 0.5 & 0.5 \\
4 & 2854.206196 & 12 & 0.5 & + & $\mathrm{e}$ & X2SIGMA & 3 & 0 & 0.5 & 0.5 \\
5 & 3777.503929 & 12 & 0.5 & + & $\mathrm{e}$ & X2SIGMA + & 4 & 0 & 0.5 & 0.5 \\
6 & 4686.660386 & 12 & 0.5 & + & $\mathrm{e}$ & X2SIGMA+ & 5 & 0 & 0.5 & 0.5 \\
7 & 5346.116382 & 12 & 0.5 & + & $\mathrm{e}$ & A2PI & 0 & 1 & 0.5 & 0.5 \\
8 & 5581.906844 & 12 & 0.5 & + & $\mathrm{e}$ & X2SIGMA+ & 6 & 0 & 0.5 & 0.5 \\
9 & 6066.934830 & 12 & 0.5 & + & $\mathrm{e}$ & A2PI & 1 & 1 & 0.5 & 0.5 \\
10 & 6463.039443 & 12 & 0.5 & + & $\mathrm{e}$ & X2SIGMA+ & 7 & 0 & 0.5 & 0.5 \\
11 & 6778.997803 & 12 & 0.5 & + & $\mathrm{e}$ & A2PI & 2 & 1 & 0.5 & 0.5 \\
12 & 7329.427637 & 12 & 0.5 & + & $\mathrm{e}$ & X2SIGMA+ & 8 & 0 & 0.5 & 0.5 \\
13 & 7483.145675 & 12 & 0.5 & + & $\mathrm{e}$ & A2PI & 3 & 1 & 0.5 & 0.5 \\
14 & 8159.170405 & 12 & 0.5 & + & $\mathrm{e}$ & A2PI & 4 & 1 & 0.5 & 0.5 \\
15 & 8201.467744 & 12 & 0.5 & + & $\mathrm{e}$ & X2SIGMA+ & 9 & 0 & 0.5 & 0.5 \\
16 & 8857.266385 & 12 & 0.5 & + & $\mathrm{e}$ & A2PI & 5 & 1 & 0.5 & 0.5 \\
17 & 9029.150380 & 12 & 0.5 & + & $\mathrm{e}$ & X2SIGMA+ & 10 & 0 & 0.5 & 0.5 \\
18 & 9535.195842 & 12 & 0.5 & + & $\mathrm{e}$ & A2PI & 6 & 1 & 0.5 & 0.5 \\
19 & 9854.882567 & 12 & 0.5 & + & $\mathrm{e}$ & X2SIGMA+ & 11 & 0 & 0.5 & 0.5 \\
20 & 10204.019475 & 12 & 0.5 & + & $\mathrm{e}$ & A2PI & 7 & 1 & 0.5 & 0.5 \\
21 & 10667.668381 & 12 & 0.5 & + & $\mathrm{e}$ & X2SIGMA+ & 12 & 0 & 0.5 & 0.5 \\
22 & 10864.560220 & 12 & 0.5 & + & $\mathrm{e}$ & A2PI & 8 & 1 & 0.5 & 0.5 \\
23 & 11464.897083 & 12 & 0.5 & + & $\mathrm{e}$ & X2SIGMA+ & 13 & 0 & 0.5 & 0.5 \\
24 & 11519.212123 & 12 & 0.5 & + & $\mathrm{e}$ & A2PI & 9 & 1 & 0.5 & 0.5 \\
25 & 12156.974798 & 12 & 0.5 & + & $\mathrm{e}$ & A2PI & 10 & 1 & 0.5 & 0.5 \\
26 & 12257.694655 & 12 & 0.5 & + & $\mathrm{e}$ & X2SIGMA+ & 14 & 0 & 0.5 & 0.5 \\
27 & 12793.671660 & 12 & 0.5 & + & $\mathrm{e}$ & A2PI & 11 & 1 & 0.5 & 0.5 \\
28 & 13030.412255 & 12 & 0.5 & + & $\mathrm{e}$ & X2SIGMA+ & 15 & 0 & 0.5 & 0.5 \\
29 & 13421.583651 & 12 & 0.5 & + & $\mathrm{e}$ & A2PI & 12 & 1 & 0.5 & 0.5 \\
30 & 13790.933964 & 12 & 0.5 & + & $\mathrm{e}$ & X2SIGMA+ & 16 & 0 & 0.5 & 0.5 \\
\hline
\end{tabular}

$n$ : State counting number.

$\tilde{E}$ : State energy in $\mathrm{cm}^{-1}$.

$g$ : State degeneracy.

$J$ : Total angular momentum.

$+/-$ : Total parity.

$e / f$ : Rotationless parity.

State: Electronic state label.

$v$ : State vibrational quantum number.

$\Lambda$ : Absolute value of $\Lambda$ (projection of the electronic angular momentum).

$\Sigma$ : Absolute value of $\Sigma$ (projection of the electronic spin).

$\Omega$ : Absolute value of $\Omega=\Lambda+\Sigma$ (projeetion of the total angular momentum). 
Table 3: Sample extracts from the output 'Transition' files produced by Duo for the ${ }^{27} \mathrm{Al}^{16} \mathrm{O}$ molecule [48].

\begin{tabular}{cccr}
\hline \hline$n_{f}$ & $n_{i}$ & $A_{f i}$ & \multicolumn{1}{c}{$\tilde{v}_{f i}$} \\
\hline \hline 173 & 1 & $4.2062 \mathrm{E}-06$ & 1.274220 \\
174 & 1 & $1.3462 \mathrm{E}-02$ & 966.699387 \\
175 & 1 & $1.3856 \mathrm{E}-02$ & 1918.099262 \\
176 & 1 & $9.7421 \mathrm{E}-03$ & 2855.450672 \\
177 & 1 & $1.2511 \mathrm{E}-06$ & 3778.740157 \\
178 & 1 & $1.1544 \mathrm{E}-02$ & 4687.891466 \\
179 & 1 & $6.7561 \mathrm{E}+02$ & 5346.110326 \\
180 & 1 & $4.1345 \mathrm{E}+00$ & 5583.130067 \\
181 & 1 & $2.4676 \mathrm{E}+03$ & 6066.924557 \\
182 & 1 & $3.5289 \mathrm{E}+01$ & 6464.257469 \\
183 & 1 & $4.6960 \mathrm{E}+03$ & 6778.981670 \\
184 & 1 & $1.9771 \mathrm{E}+02$ & 7330.641321 \\
185 & 1 & $6.1540 \mathrm{E}+03$ & 7483.122722 \\
186 & 1 & $4.8062 \mathrm{E}+03$ & 8159.737396 \\
187 & 1 & $1.9401 \mathrm{E}+03$ & 8202.080179 \\
\hline$n_{f}:$ Upper state counting number. \\
$n_{i}:$ Lower state counting number. \\
$A_{f i}:$ Einstein-A coefficient in s ${ }^{-1}$. \\
$\tilde{v}_{f i}:$ Transition wavenumber in $\mathrm{cm}^{-1}$ (optional).
\end{tabular}




\section{Inverse problem}

The inverse problem consists in finding the potential energy and coupling curves which best reproduce a given set of energy levels, $E_{i}^{\text {(obs) }}$, or frequencies (i.e., differences between energy levels), typically extracted from experiment. In the following we will call this optimization process empirical refinement.

\subsection{Implementation}

The refinement problem can be formulated as a non-linear least-squares problem where one seeks to minimize the objective function [120]:

$$
F=\sum_{i}\left[E_{i}^{(\mathrm{obs})}-E_{i}^{(\mathrm{calc})}\left(a_{n}\right)\right]^{2} w_{i}
$$

where $E_{i}^{\text {(calc) }}\left(a_{n}\right)$ are the calculated energies or frequencies and implicitly depend on the parameters $a_{1}, a_{2}, \ldots$ defining the potential and coupling curves. The $w_{i}>0$ are weighting factor assigned to each value and may be chosen as $1 / \sigma_{i}^{2}$ where $\sigma_{i}$ is the experimental uncertainty of $E_{i}^{(\mathrm{obs})}$. The input weights are automatically renormalized by Duo so that $\sum_{i} w_{i}=1$.

Duo uses the non-linear conjugate gradient method for the optimization; in particular, the linearized least-square problem is solved by default using the LAPACK subroutine DGELSS, although the alternative built-in subroutine LINUR is also available. For each curve appearing in Duo it is possible to specify if any given parameter should be refined (fitted) or should be kept fixed to the value given in the input file. The first derivatives with respect to the fitting parameters $a_{n}$ required for the non-linear least squares are computed using finite differences with a step size $\Delta a_{n}$ taken as $0.1 \%$ of the initial values $a_{n}$ or 0.001 if $a_{n}$ is initially zero.

\subsection{Constrained minimization}

In order to avoid unphysical behaviour and also to avoid problems when the amount of experimental data provided is insufficient for determining all the parameters, the shapes of the curves can be contrained to be as close as possible to some reference curves provided in the input (typically ab initio ones) [28, 121-123]. This is done by including into the fitting objective function not only differences of the computed energy levels but also differences between the refined curves $V^{\lambda \text {,(calc) }}$ and the reference ones $V^{\lambda, \text { (ref) }}$ as follows:

$$
F=\sum_{i}\left(E_{i}^{(\mathrm{obs})}-E_{i}^{(\mathrm{calc})}\right)^{2} w_{i}^{\mathrm{en}}+\sum_{\lambda} d_{\lambda} \sum_{k}\left(V_{k}^{\lambda,(\mathrm{ref})}-V_{k}^{\lambda,(\mathrm{calc})}\right)^{2} w_{k}^{\lambda},
$$

where $\lambda$ refers to the $\lambda$-th curve, $k$ counts over the grid points, $w_{i}^{\lambda}$ are the corresponding weight factors of the individual points normalized to one and $d_{\lambda}$ are 
further weight factors defining the relative importance of the corresponding curve. The weights in Eq. (45) are normalized as follows:

$$
\sum_{i} w_{i}^{\mathrm{en}}+\sum_{\lambda} \sum_{k} d_{\lambda} w_{k}^{\lambda}=1
$$

When minimizing the functional given by Eq. (45) it is important to control the correctness of the match between the experimental and theoretical levels as they appear in the corresponding observed ('obs.') and calculated ('calc.') lists. It is typical in complex fits involving close-lying electronic states that the order of the computed energy levels in a $(J, \tau)$ block changes during the empirical refinement. In order to follow these changes and update the positions of the experimental values in the fitting set, we use the quantum numbers to identify the corresponding quantities by locking to their initial values. Since the experimentally assigned quantum numbers may not agree with the ones used by Duo - which are based on Hund's case $a$ ) — each experimental energy level (or frequency) $E_{i}^{(\mathrm{obs})}$ is automatically labelled by Duo with the following quantum numbers: $J$, parity $\tau( \pm)$, 'state', $v,|\Lambda|,|\Sigma|$ and $|\Omega|$; this set of six quantum numbers is then used for matching with a calculated counterpart $E_{i}^{(\mathrm{calc})}$. Note that only the absolute values of $\Lambda$, $\Sigma$ and $\Omega$ are used for this purpose, as their sign is undefined in the general case.

\subsubsection{Morphing}

The curves to be refined can also be 'morphed' [47, 124, 125] by scaling them by a function $H(r)$, so that the refined function $F(r)$ at a given grid point $r_{i}$ is given by

$$
F\left(r_{i}\right)=H\left(r_{i}\right) F^{\text {initial }}\left(r_{i}\right),
$$

where $F^{\text {ai }}\left(r_{i}\right)$ is initial function specified in the input file (e.g., obtained by ab initio methods). For this kind of empirical refinement the curves $F^{\text {ai }}$ do not necessarily have to be specified by a parametrised analytical form but can also be provided as a spline interpolant as described in Section 5.2.

The morphing function $H(r)$ is typically represented by a simple polynomial, see Patrascu et al. [47] for an example. The morphing approach is an alternative way of constraining the refined properties to the reference curve and is especially useful when experimental information is sparse.

\section{Types of functional forms}

\subsection{Analytical representations}

A number of functional forms are currently available in Duo to specify $r$ dependent curves (e.g., potential energy curves, dipole moment curves) as parametrised analytical functions. In the following $T_{\mathrm{e}}$ will represent the value of the potential at the equilibrium geometry $r_{\mathrm{e}}$. 
1. Expansion in Dunham variables [126]:

$$
V(r)=T_{\mathrm{e}}+a_{0} y_{\mathrm{D}}^{2}(r)\left(1+\sum_{i \geq 1} a_{i} y_{\mathrm{D}}^{i}(r)\right),
$$

where

$$
y_{\mathrm{D}}(r)=\frac{r-r_{\mathrm{e}}}{r_{\mathrm{e}}} .
$$

2. Taylor polynomial expansion:

$$
V(r)=T_{\mathrm{e}}+\sum_{i \geq 0} a_{i}\left(r-r_{\mathrm{e}}\right)^{i}
$$

3. Simons-Parr-Finlan (SPF) [127, 128] expansion:

$$
V(r)=T_{\mathrm{e}}+a_{0} y_{\mathrm{SPF}}^{2}(r)\left(1+\sum_{i \geq 1} a_{i} y_{\mathrm{SPF}}^{i}(r)\right),
$$

where

$$
y_{\mathrm{SPF}}(r)=\frac{r-r_{\mathrm{e}}}{r} .
$$

4. Murrell-Sorbie (MS) [129]:

$$
V(r)=T_{\mathrm{e}}+\left(A_{\mathrm{e}}-T_{\mathrm{e}}\right) e^{-a\left(r-r_{\mathrm{e}}\right)}\left(1+\sum_{i \geq 1} a_{i}\left(r-r_{\mathrm{e}}\right)^{i}\right),
$$

where $A_{\mathrm{e}}$ is the asymptote of $V(r)$ at $r \rightarrow+\infty$ relative to

$T_{\mathrm{e}}$ of the lowest electronic state, related to the commonly used dissociation energy of the given electronic state $D_{\mathrm{e}}=A_{\mathrm{e}}-T_{\mathrm{e}}$.

5. Chebyshev polynomial expansion $[130,131]$ :

$$
V(r)=\left[T_{\mathrm{e}}+D_{\mathrm{e}}\right]-\frac{\sum_{i=0} a_{i} T_{k}\left(y_{p}\right)}{1+\left(r / r_{\mathrm{ref}}\right)^{n}},
$$

in which $n$ is a positive integer and $T_{k}(y)$ are the Chebyshev polynomials of the first kind defined in terms of the reduced variable $y_{p}(r) \in[-1,1]$ :

$$
y_{p}\left(r ; r_{\text {min }}, r_{\text {ref }}\right)=\frac{r^{p}-r_{\text {ref }}^{p}}{r^{p}+r_{\text {ref }}^{p}-2 r_{\text {min }}^{p}}
$$

with $p$ as the fixed parameter. This form guarantees the correct long-range (LR) behaviour at $r \rightarrow \infty$ :

$$
V(r) \rightarrow u_{\mathrm{LR}}(r)=\sum_{n} \frac{C_{n}}{r^{n}}
$$

where the $C_{n}$ are the long-range coefficients. 
6. Perturbed Morse Oscillator (PMO) [132-137]:

$$
V(r)=T_{\mathrm{e}}+\left(A_{\mathrm{e}}-T_{\mathrm{e}}\right) y_{\mathrm{M}}^{2}+\sum_{i=1} a_{i} y_{\mathrm{M}}^{i+2},
$$

where

$$
y_{\mathrm{M}}=1-\exp \left(-\beta\left(r-r_{\mathrm{e}}\right) .\right.
$$

When $a_{i}=0$ the form reduces to the Morse potential, otherwise the asymptotic value of the potential is $A_{\mathrm{e}}+\sum_{i} a_{i}$.

7. Extended Morse Oscillator (EMO) [138, 139]:

$$
V(r)=T_{\mathrm{e}}+\left(A_{\mathrm{e}}-T_{\mathrm{e}}\right)\left(1-\exp \left\{-\beta_{\mathrm{EMO}}(r)\left(r-r_{\mathrm{e}}\right)\right\}\right)^{2},
$$

which has the form of a Morse potential with a exponential tail and a distancedependent exponent coefficient

$$
\beta_{\mathrm{EMO}}(r)=\sum_{i=0} a_{i} y_{p}^{\mathrm{eq}}(r)^{i}
$$

expressed as a power series in the reduced variable [140]:

$$
y_{p}^{\mathrm{eq}}(r)=\frac{r^{p}-r_{\mathrm{e}}^{p}}{r^{p}+r_{\mathrm{e}}^{p}} .
$$

8. Morse Long-Range (MLR) function [23, 34, 139, 141]:

$$
V(r)=T_{\mathrm{e}}+\left(A_{\mathrm{e}}-T_{\mathrm{e}}\right)\left(1-\frac{u_{\mathrm{LR}}(r)}{u_{\mathrm{LR}}\left(r_{e}\right)} \exp \left\{-\beta_{\mathrm{MLR}}(r) y_{p}^{\mathrm{eq}}(r)\right\}\right)^{2},
$$

where the radial variable $y_{p}^{\mathrm{eq}}$ in the exponent is given by Eq. (57), the longrange potential $u_{\mathrm{LR}}(r)$ by Eq. (53) while the exponent coefficient function

$$
\beta_{\mathrm{MLR}}(r)=y_{p}^{\mathrm{ref}}(r) \beta_{\infty}+\left[1-y_{p}^{\mathrm{ref}}(r)\right] \sum_{i=0} a_{i}\left[y_{q}^{\mathrm{ref}}(r)\right]^{i}
$$

is defined in terms of two radial variables which are similar to $y_{p}^{\mathrm{eq}}$, but are defined with respect to a different expansion centre $r_{\text {ref }}$, and involve two different powers, $p$ and $q$. The above definition of the function $\beta_{\mathrm{MLR}}(r)$ means that:

$$
\beta_{\mathrm{MLR}}(r \rightarrow \infty) \equiv \beta_{\infty}=\ln \left[2 D_{\mathrm{e}} / u_{\mathrm{LR}}\left(r_{\mathrm{e}}\right)\right] .
$$

9. Šurkus-polynomial expansion [140]:

$$
V(r)=T_{\mathrm{e}}+\left(1-y_{p}^{\mathrm{eq}}\right) \sum_{i \geq 0} a_{i}\left[y_{p}^{\mathrm{eq}}\right]^{i}+y_{p}^{\mathrm{eq}} a_{\mathrm{inf}},
$$

where $y_{p}^{\mathrm{eq}}$ is the Šurkus variable (57) and $a_{\mathrm{inf}}$ is the asymptote of the potential at $r \rightarrow \infty$. 
10. Šurkus-polynomial expansion with a damping function [140]:

$$
V(r)=T_{\mathrm{e}}+\left[\left(1-y_{p}^{\mathrm{eq}}\right) \sum_{i \geq 0} a_{i}\left[y_{p}^{\mathrm{eq}}\right]^{i}+y_{p}^{\mathrm{eq}} a_{\mathrm{inf}}\right] f^{\mathrm{damp}}+t^{\mathrm{damp}}\left(1-f^{\mathrm{damp}}\right),
$$

where the damping function is defined by

$$
f^{\mathrm{damp}}=1-\tanh \left[\alpha\left(r-r_{0}\right)\right]
$$

and $t^{\mathrm{damp}}, r_{0}$ and $\alpha$ are parameters.

\subsection{Numerical representations}

Any $r$-dependent curve $F(r)$ can be specified as a list of data points $\left\{r_{k}, F\left(r_{k}\right)\right\}, k=$ $1, \ldots, N_{p}$ for a range of geometries. Duo will then automatically interpolate or extrapolate the data points whenever necessary. The interpolation within the specified range is performed either by using cubic or quintic splines. More specifically, Duo uses natural cubic splines in the form given in Ref. [142] or natural quintic splines based on an adaptation of the routine QUINAT [143, 144]. Quintic splines are used by default, as they generally provide quicker convergence of the interpolant with respect to the number of points given; however, they may lead to spurious oscillations between the data points, especially for non-uniform grids. The number of data points should be $\geq 4$ for cubic splines and $\geq 6$ for quintic splines. It is sometimes useful to interpolate a transformed set, such as $\left(r_{i}, r_{i}^{2} F_{i}\right)$ or $\left(1 / r_{i}, F_{i}\right)$, see, for example, Refs. [145, 146] or the discussion by Lodi [147]; this feature is not yet implemented in Duo.

When necessary curves are extrapolated at short range (i.e., in the interval $\left.\left[r_{\min }, r_{1}\right]\right)$ using one of the following functional forms:

$$
\begin{aligned}
& f_{1}(r)=A+B / r \\
& f_{2}(r)=A r+B r^{2}, \\
& f_{3}(r)=A+B r,
\end{aligned}
$$

where the constants $A$ and $B$ are found by requiring that the functions $f_{i}$ go through the first two data points. By default the functional form $f_{1}$ is used for potential energy curves, form $f_{2}$ is used for the transition dipole moments and form $f_{3}$ (linear extrapolation) for all other curves (for example spin-orbit couplings). The default choices should be appropriate in most cases.

Similarly, whenever necessary curves are extrapolated at long range (i.e., in the interval $\left.\left[r_{N_{k}}, r_{\max }\right]\right)$ by fitting the last two data points to

$$
\begin{aligned}
& f_{4}(r)=A+B / r^{6} \\
& f_{5}(r)=A+B r \\
& f_{6}(r)=A / r^{2}+B / r^{3} .
\end{aligned}
$$


These functional forms were chosen to describe the behaviour at long ranges of the potential energy curves $\left[f_{4}(r)\right]$, of the curves corresponding to the electronic angular momenta $L_{x}(r), L_{y}(r), L_{ \pm}(r)\left[f_{5}(r)\right]$; for all other cases (including transition dipole moment) the functional form $f_{6}$ is assumed. Note that form $f_{4}(r)$ is appropriate in many but by no means in all cases, see ref. [148] for details on the the asymptotic behaviour of the potential energy curves at large $r$.

Similarly, Ref. [149] discusses the asymptotic forms of molecular (diagonal) dipole functions and states the correct limits have the form $A+B r^{m}$ with $m=3$ or 5 for $r \rightarrow 0$ and $A+B / r^{m}$ with $m=4$ or 7 for $r \rightarrow \infty$ so that our extrapolation forms do not have the correct asymptotic forms. Nevertheless usually extrapolation is performed quite far from the aymptotic region so that using the theoretically correct form is not required nor, indeed, beneficial in such cases.

It should be noted that the extrapolation procedures described introduce a small discontinuity in the first derivatives at the switching points $r_{1}$ or $r_{N_{p}}$. In some situations these artifacts could become important, e.g. for very loosely bound states such as those discussed in section 2.2. In such cases it is recommeded to use an analytical representation for the potential with an appropriate long-range behaviour, e.g. the Morse long-range form given by Eq. (58).

\section{Program inputs and structure}

The Duo calculation set up is specified by an input file in the plain text (ASCII) format. The input contains the specifications of the relevant terms of the Hamiltonian (i.e., the potential energy and coupling curves), dipole moment curves as well as options determining the method used for the solution, convergence thresholds etc. Different couplings, corrections or tasks are switched on by adding the corresponding section to the input file, i.e. without any alternation of the code. The input is controlled by keywords and makes use of Stone's input parser [150]. All keywords and options are fully documented in the manual provided along with the source code. The structure of the program is illustrated in Fig. 4.

In addition to the program source code and the manual, we also provide makefiles for various Fortran 2003 compilers as well as a set of four examples with sample inputs and corresponding outputs. These examples comprise (a) a very simple test based on numerical solution of a single Morse potential, (b) a fit of a single ${ }^{2} \Pi$ state to observed energies based on a recent study of the PS molecule [151], (c) a fit of a single ${ }^{3} \Sigma^{-}$based on a recent study of the PH molecule [151], and (d) calculation of the spectrum of $\mathrm{ScH}$ involving 6 electronic states based on recent study [49]; the output files in this case are given in ExoMol format [118]. 


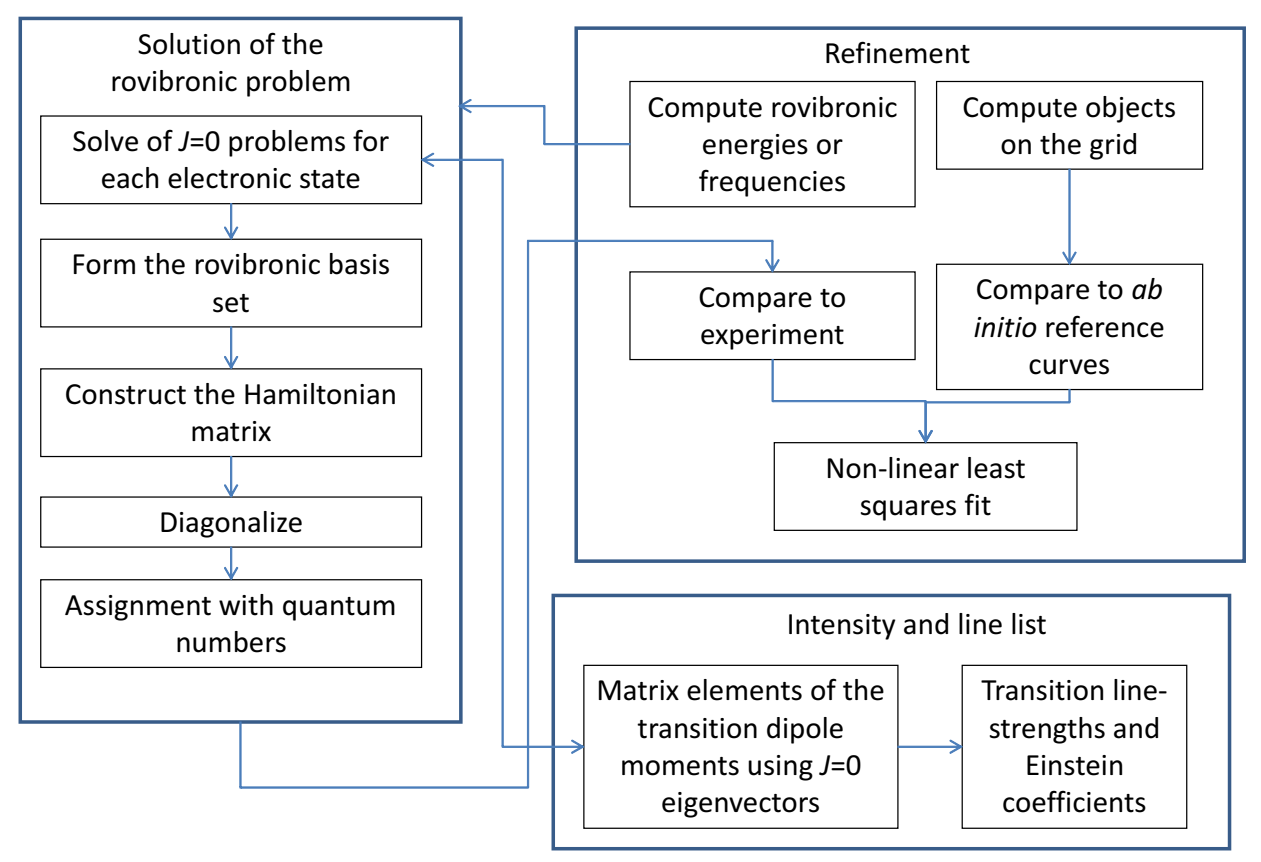

Figure 4: Duo program structure.

These examples only consider low levels of rotational excitation, $J$, to make them fast to run.

\section{Conclusion}

Duo is a highly flexible code for solving the nuclear motion problem for diatomic molecules with non-adiabatically coupled electronic states. It can simulate pure rotational, ro-vibrational and rovibronic spectra using an entirely $a b$ initio input from electronic structure calculations or semi-empirical data. The latter can also be obtained within Duo by fitting to experimental data. Duo is currently being further developed and extensively used to study a number of diatomic species [47, 49-51, 152] as part of the ExoMol project [46]. This project is primarily interested in hot molecules, but Duo should be equally useful for studying ultracold diatomic molecules.

\section{Acknowledgements}

This work is supported by ERC Advanced Investigator Project 267219 and by EPSRC Collaborative Computational Project Q (CCPQ) on Quantum Dynamics. Duo uses the Fortran 90 input parsing module input.f90 supplied by Anthony J. Stone, which is gratefully acknowledged. We also thank members of the ExoMol 
project Laura McKemmish, Andrei Patrascu, Marcus Vasilios, Frances Sinden, Thomas Fraser, Audra Blissett, Usama Asari, Pawel Jagoda, Laxmi Prajapat and Maire Gorman for for their help with testing Duo. AVS would like to thank the University College London for hospitality during his visits in 2012-13. The work in MSU was partially supported by the RFBR grant No. 15-03-03302-a.

\section{References}

[1] L. S. Cederbaum, Born-Oppenheimer and beyond, Advanced Series in Physical Chemistry: Conical Intersections Electronic Structure, Dynamics and Spectroscopy (W. Domcke, D. R. Yarkony and H. Köppel eds. 15 (2004) 3.

[2] F. A. Berezin, M. A. Shubin, The Schrödinger equation, Kluwer Academic Publishers, 1991.

[3] B. Simon, Schrödinger operators in the twentieth century, J. Math. Phys. 41 (2000) 3523-3555.

[4] J. M. Blatt, Practical points concerning the solution of the Schrödinger equation, J. Comp. Phys. 1 (382) 1967.

[5] B. W. Shore, Solving the radial Schrödinger equation by using cubic spline basis functions, J. Chem. Phys. 58 (1973) 3855.

[6] B. R. Johnson, New numerical methods applied to solving the onedimensional eigenvalue problem, J. Chem. Phys. 67 (1977) 4086.

[7] B. R. Johnson, The renormalized Numerov method applied to calculating bound states of the coupled-channel Schroedinger equation, J. Chem. Phys. 69 (1978) 4678.

[8] H. J. Korsch, H. Laurent, Milne $\not \varnothing s$ differential equation and numerical solutions of the Schrödinger equation I. Bound-state energies for single and double-minimum potentials, J. Phys. B: At. Mol. Phys. 14 (1981) 4213.

[9] R. Guardiola, J. Ros, On the Numerical Integration of the Schrödinger Equation in the Finite-Difference Schemes, J. Comp. Phys. 45 (1982) 374.

[10] R. Guardiola, J. Ros, On the Numerical Integration of the Schrödinger Equation: numerical tests, J. Comp. Phys. 45 (1982) 390.

[11] A. V. Stolyarov, N. E. Kuz'menko, Solution of the Radial Schrödinger Equation by a Modified "Shooting" Method, Czech. J. Phys. B 37 (1987) 529. 
[12] B. Lindberg, A new efficient method for calculation of energy eigenvalues and eigenstates of the one-dimensional Schrödinger equation, J. Chem. Phys. 88 (1988) 3805.

[13] C. C. Marston, G. G. Balint-Kurti, The Fourier grid Hamiltonian method for bound state eigenvalues and eigenfunctions, J. Chem. Phys. 91 (1989) 3571 .

[14] A. V. Abarenov, A. V. Stolyarov, The phase formalism for the onedimensional eigenvalue problem and its relation with quantum BornSommerfeld rule, J. Phys. B: At. Mol. Opt. Phys. 23 (1990) 2419-2426.

[15] R. V. J. Garza, A. Vela, Strongly convergent method to solve onedimensional quantum problems, Phys. Rev. E 53 (1996) 1954.

[16] R. V. J. Garza, A. Vela, Reply to "Comment on 'Strongly convergent method to solve one-dimensional quantum problems' ", Phys. Rev. E 56 (1997) 1954.

[17] H. Ishikawa, An accurate method for numerical calculations in quantum mechanics, J. Phys. A: Math. Gen. 35 (2002) 4453.

[18] T. Utsumi, T. Yabe, J. Koga, T. Aoki, M. Sekine, Accurate basis set by the CIP method for the solutions of the Schrödinger equation, Comput. Phys. Commun. 157 (2004) 121.

[19] Z. Wang, Y. Ge, Y. Dai, D. Zhao, A Mathematica program for the twostep twelfth-order method with multi-derivative for the numerical solution of a one-dimensional Schrdinger equation, Comput. Phys. Commun. 160 (2004) 23.

[20] J. W. Cooley, An Improved eigenvalue corrector formula for solving the Schrödinger equation for central fields, Math. Comp. 15 (1961) 363-374, doi:http://dx.doi.org/10.1090/S0025-5718-1961-0129566-X.

[21] J. K. Cashion, Testing of diatomic potential energy functions by numerical methods, J. Chem. Phys. 39 (1963) 1872.

[22] B. V. Noumerov, A method of extrapolation of perturbations, Mon. Not. R. Astron. Soc. 84 (1924) 592-602, doi:10.1093/mnras/84.8.592.

[23] R. J. Le Roy, LEVEL 8.0 A Computer Program for Solving the Radial Schrödinger Equation for Bound and Quasibound Levels, University of Waterloo Chemical Physics Research Report CP-663, http://leroy.uwaterloo.ca/programs/, 2007. 
[24] J. Karkowski, Inverse Problems in Quantum Chemistry, Int. J. Quant. Chem. 109 (2009) 2456-2463.

[25] T. Weymuth, M. Reiher, Inverse Quantum Chemistry: Concepts and Strategies for Rational Compound Design, Int. J. Quant. Chem. 114 (2014) 823837.

[26] W. M. Kosman, J. Hinze, Inverse pertubation analysis: improving the accuracy of potential energy curves, J. Mol. Spectrosc. 56 (1975) 93-103.

[27] A. Pashov, W. Jastrzebski, P. Kowalczyk, Construction of potential curves for diatomic molecular states by the IPA method, Comput. Phys. Commun. 128 (2000) 622-634.

[28] T. Szidarovszky, A. G. Császár, Grid-Based Empirical Improvement of Molecular Potential Energy Surfaces, J. Phys. Chem. A 118 (2014) 62566265, doi:10.1021/jp504348f.

[29] R. J. Le Roy, DPotFit 1.1 A Computer Program for Fitting Diatomic Molecule Spectral Data to Potential Energy Functions, University of Waterloo Chemical Physics Research Report CP-662R, http://leroy. uwaterloo.ca/programs/, 2006.

[30] J. A. Coxon, P. G. Hajigeorgiou, Born-Oppenheimer Breakdown in the Ground-State of Carbon-Monoxide - a Direct Reduction of Spectroscopic Line Positions to Analytical Radial Hamiltonian Operators, Can. J. Phys. 70 (1992) 40-54.

[31] J. A. Coxon, P. G. Hajigeorgiou, Experimental Born-Oppenheimer Potential for the $\mathrm{X}^{1} \Sigma^{+}$Ground State of $\mathrm{HeH}^{+}$: Comparison with the Ab Initio Potential, J. Mol. Spectrosc. 193 (1999) 306-318.

[32] J. A. Coxon, P. G. Hajigeorgiou, Direct potential fit analysis of the X-1 Sigma(+) ground state of CO, J. Chem. Phys. 121 (2004) 2992-3008, doi: $10.1063 / 1.1768167$.

[33] R. J. Le Roy, Algebraic vs. numerical methods for analysing diatomic spectral data: a resolution of discrepancies, J. Mol. Spectrosc. 228 (2004) 92104, doi:10.1016/j.jms.2004.03.022.

[34] R. J. Le Roy, C. C. Haugen, J. Tao, H. Li, Long-range damping functions improve the short-range behaviour of $\phi \mathrm{MLR} \phi \varnothing$ potential energy functions, Mol. Phys. 109 (2011) 435-446. 
[35] V. V. Meshkov, A. V. Stolyarov, M. C. Heaven, C. C. Haugen, R. J. Le Roy, Direct-potential-fit analyses yield improved empirical potentials for the ground X1£g+ state of Be2, J. Chem. Phys. 140 (2014) 064315.

[36] S.-D. Walji, K. Sentjens, R. J. L. Roy, Dissociation Energies and Potential Energy Functions for the Ground X and Avoided-Crossing A States of $\mathrm{NaH}$, J. Chem. Phys. Submitted.

[37] A. Carrington, C. A. Leach, A. J. Marr, A. M. Shaw, M. R. Viant, J. M. Hutson, M. M. Law, Microwave spectroscopy and interaction potential of the long $\phi$ range He...Ar ${ }^{+}$ion, J. Chem. Phys. 102 (1995) 2379-2403, doi: http://dx.doi.org/10.1063/1.468670.

[38] M. Tamanis, R. Ferber, A. Zaitsevskii, E. A. Pazyuk, A. V. Stolyarov, H. Chen, J. Qi, H. Wang, W. C. Stwalley, High resolution spectroscopy and channel-coupling treatment of the A(1)Sigma(+)-b(3)Pi complex of NaRb, J. Chem. Phys. 117 (2002) 7980-7988.

[39] T. Bergeman, C. E. Fellows, R. F. Gutterres, C. Amiot, Analysis of strongly coupled electronic states in diatomic molecules: Low-lying excited states of RbCs, Phys. Rev. A 67 (2003) 050501(R).

[40] V. V. Meshkov, A. Zaitsevskii, E. A. Pazyuk, A. V. Stolyarov, R. Bruhl, , D. Zimmermann, Direct deperturbation analysis of the $A^{2} \Pi-B^{2} \Sigma^{+}$complex of ${ }^{7,6}$ LiAr isotopomers, J. Chem. Phys. 123 (2005) 204307.

[41] J. M. Hutson, E. Tiesinga, P. S. Julienne, Avoided crossings between bound states of ultracold cesium dimers, Phys. Rev. A 78 (2008) 052703, doi: 10.1103/PhysRevA.78.052703.

[42] P. Zhang, H. R. Sadeghpour, A. Dalgarno, Structure and spectroscopy of ground and excited states of LiYb, J. Chem. Phys. 133 (2010) 044306, doi: $10.1063 / 1.3462245$.

[43] G. Gopakumar, M. Abe, M. Hada, M. Kajita, Ab initio study of ground and excited states of ( $\mathrm{LiCa}$ )-Li-6-Ca-40 and (LiSr)-Li-6-Sr-88 molecules, J. Chem. Phys. 138 (2013) 194307, doi:10.1063/1.4804622.

[44] J. S. A. Brooke, R. S. Ram, C. M. Western, G. Li, D. W. Schwenke, P. F. Bernath, Einstein A Coefficients and Oscillator Strengths for the $A^{2} \Pi-$ $X^{2} \Sigma^{+}$(Red) and $B^{2} \Sigma^{+}-X^{2} \Sigma^{+}$(Violet) Systems and Rovibrational Transitions in the $X^{2} \Sigma^{+}$State of CN, Astrophys. J. Suppl. 210 (2014) 23, doi: $10.1088 / 0067-0049 / 210 / 2 / 23$. 
[45] J. M. Hutson, Coupled channel methods for solving the bound-state Schrödinger equation, Comput. Phys. Commun. 84 (1994) 1-18.

[46] J. Tennyson, S. N. Yurchenko, ExoMol: molecular line lists for exoplanet and other atmospheres, Mon. Not. R. Astron. Soc. 425 (2012) 21-33.

[47] A. T. Patrascu, C. Hill, J. Tennyson, S. N. Yurchenko, Study of the electronic and rovibronic structure of the $X^{2} \Sigma^{+} A^{2} \Pi$ and $B^{2} \Sigma^{+}$states of AlO, J. Chem. Phys. 141 (2014) 144312, doi:http://dx.doi.org/10.1063/1.4897484.

[48] A. T. Patrascu, J. Tennyson, S. N. Yurchenko, ExoMol molecular linelists: VIII: The spectrum of AlO, Mon. Not. R. Astron. Soc. 449 (2015) 36133619, doi:10.1093/mnras/stv507.

[49] L. Lodi, S. N. Yurchenko, J. Tennyson, The calculated rovibronic spectrum of scandium hydride, ScH, Mol. Phys. 113 (2015) 1559-1575, doi: 10.1080/00268976.2015.1029996.

[50] S. N. Yurchenko, A. Blissett, U. Asari, M. Vasilios, C. Hill, J. Tennyson, ExoMol Molecular linelists - XIV. The spectrum of $\mathrm{CaO}$, Mon. Not. R. Astron. Soc. .

[51] L. K. McKemmish, S. N. Yurchenko, J. Tennyson, ExoMol Molecular linelists - XV. The spectrum of Vanadium Oxide, Mon. Not. R. Astron. Soc. (2015) in preparation.

[52] J. Tennyson, L. Lodi, L. K. McKemmish, S. N. Yurchenko, The ab initio calculation of spectra of open shell diatomic molecules, J. Phys. B: At. Mol. Opt. Phys. Topical Review.

[53] R. Islampour, M. Miralinaghi, An extensive study of transformation of the diatomics Hamiltonian Operator from laboratory to body-fixed frame, Int. J. Quant. Chem. 115 (2015) 510-522.

[54] B. T. Sutcliffe, The separation of electronic and nuclear motion in the diatomic molecule, Theor. Chem. Acc. 118 (2007) 563-571.

[55] H. Kato, Energy-Levels and Line-Intensities of Diatomic-Molecules - Application to Alkali-Metal Molecules, Bull. Chem. Soc. Japan 66 (1993) 3203-3234, doi:10.1246/bcsj.66.3203.

[56] R. Pack, J. Hirschfelder, Separation of rotational coordinates from the $\mathrm{N}$ electron diatomic Schrödinger equation, J. Chem. Phys. 49 (1968) 40094020 . 
[57] P. R. Bunker, The electronic isotope shift in diatomic molecules and the partial breakdown of the Born-Oppenheimer approximation, J. Mol. Spectrosc. 28 (1968) 422-443.

[58] W. Kutzelnigg, The adiabatic approximation. 1. The physical background of the Born-Handy ansatz, Mol. Phys. 90 (1997) 909-916.

[59] J. M. Hutson, B. J. Howard, A new approach to perturbation theory for breakdown of the Born-Oppenheimer approximation, Mol. Phys. 41 (1980) 1113.

[60] D. W. Schwenke, A unified derivation of Hamiltonian and optical transition matrix elements for open shell diatomic and polyatomic molecules using transformation tools of modern quantum mechanics, J. Chem. Phys. 142 (2015) 144107, doi:10.1063/1.4916952.

[61] E. A. Colbourn, F. D. Wayne, The values of $\left\langle L^{2}\right\rangle$ in diatomic molecules: implications for adiabatic and molecular fine structure calculations, Mol. Phys. 37 (1979) 1755-1764.

[62] A. V. Stolyarov, V. I. Pupyshev, M. S. Child, Analytical approximations for adiabatic and non-adiabatic matrix elements of homonuclear diatomic Rydberg states. Application to the singlet p-complex of the hydrogen molecule, J. Phys. B: At. Mol. Opt. Phys. 30 (1997) 3077, doi: 10.1088/0953-4075/30/14/005.

[63] A. V. Stolyarov, M. S. Child, Analog of the Hellmann-Feynman theorem in multichannel quantum-defect theory, Phys. Rev. A 63 (2001) 052510, doi:10.1103/PhysRevA.63.052510.

[64] R. M. Herman, A. Asgharian, Theory of energy shifts associated with deviations from Born-Oppenheimer behavior in ${ }^{1} \Sigma$-state diatomic molecules, J. Mol. Spectrosc. 28 (1968) 422-443.

[65] T. L. Barr, D. Dee, R. R. Gilmore, Angular momentum coupling, potential curves and radiative selection rules for heavy diatomic molecules with particular reference to $\mathrm{Kr}_{2}$ and $\mathrm{Kr}_{2}{ }^{+}$, J. Quant. Spectrosc. Radiat. Transf. 15 (1975) 625-635.

[66] I. Røeggen, The inversion eigenvalues of non- $\Sigma$ states of diatomic molecules, expressed in terms of quantum numbers, Theor. Chem. Acc. 21 (1971) 398-409. 
[67] J. R. Lund, B. V. Riley, A Sine-Collocation Method for the Computation of the Eigenvalues of the Radial Schrodinger Equation, IMA J. Numer. Anal. 4 (1984) 83.

[68] D. T. Colbert, W. H. Miller, A novel discrete variable representation for quantum mechanical reactive scattering via the S-matrix Kohn method, Journal of Chemical Physics 96 (1992) 1982-1991.

[69] D. J. Tannor, Introduction to Quantum Mechanics - A time dependent perspective, University Science Books, 2007.

[70] L. N. Trefethen, J. A. C. Weideman, The Exponentially Convergent Trapezoidal Rule, SIAM Rev. 56 (2014) 385.

[71] J. A. Gallas, Some matrix elements for Morse oscillators, Phys. Rev. A 21 (1980) 1829-1833.

[72] Z. Rong, D. Cavagnar, L. Lespade, Calculation of displacement matrix elements for morse oscillators, Lecture Notes in Computer Science 2658 (350 - 356) 2003.

[73] N. J. Higham, The accuracy of floating point summation, SIAM J. Sci. Comput. 14 (1993) 783-799.

[74] E. S. Medvedev, V. V. Meshkov, A. V. Stolyarov, I. E. Gordon, Peculiarities of high-overtone transition probabilities in carbon monoxide revealed by high-precision calculation, J. Chem. Phys. 143 (2015) 154301.

[75] J. A. Coxon, T. C. Melville, Application of direct potential fitting to line position data for the $X^{1} \Sigma_{g}^{+}$and $A^{1}{ }^{1} \Sigma_{u}^{+}$states of $\mathrm{Li}_{2}$, J. Mol. Spectrosc. 235 (2006) 235-247.

[76] P. Qi, J. Bai, E. Ahmed, A. M. Lyyra, S. Kotochigova, A. J. Ross, C. Effantin, P. Zalicki, J. Vigué, G. Chawla, R. W. Field, T.-J. Whang, W. C. Stwalley, H. Knöckel, E. Tiemann, J. Shang, L. Li, T. Bergeman, New spectroscopic data, spin-orbit functions, and global analysis of data on the $A^{1} \Sigma_{u}+$ and $b^{3} \Pi_{u}$ states of $\mathrm{Na}_{2}$, J. Chem. Phys. 127 (2007) 044301.

[77] S. Falke, I. Sherstov, E. Tiemann, C. Lisdat, The $A^{1} \Sigma_{u}^{+}$state of $\mathrm{K}_{2}$ up to the dissociation limit, J. Chem. Phys. 125 (2006) 224303.

[78] E. Tiemann, H. Knöckel, Renaissance in diatomic spectroscopy, AIP Conference proceedings 1545 (2013) 79, eighth international conference on atomic and molecular data and their applications: ICAMDATA-2012. 
[79] V. V. Meshkov, A. V. Stolyarov, R. J. Le Roy, Adaptive analytical mapping procedure for efficiently solving the radial Schrödinger equation, Phys. Rev. A 78 (2008) 052510.

[80] R. J. Le Roy, R. B. Bernstein, Shape Resonances and Rotationally Predissociating Levels: The Atomic Collision Time $\measuredangle$ Delay Functions and Quasibound Level Properties of $\mathrm{H}_{2}\left(X^{1} \Sigma_{g}^{+}\right)$, J. Chem. Phys. 54 (5114) 1971.

[81] R. J. Le Roy, W. Liu, Energies and widths of quasibound levels (orbiting resonances) for spherical potentials, J. Chem. Phys. 69 (1978) 3622.

[82] J. N. L. Connor, A. D. Smith, Uniform semi-classical calculation of resonance energies and widths near a barrier maximum, Mol. Phys. 43 (1981) 397-414.

[83] J. D. Pryce, Efficient, reliable computation of resonances of the onedimensional Schrödinger equation, J. Comp. Phys. 112 (1994) 234-246.

[84] U. V. Riss, H. D. Meyer, Calculation of resonance energies and widths using the complex absorbing potential method, J. Phys. B: At. Mol. Phys. 26 (1993) 4503-4536.

[85] M. Č́žek, J. Horáček, J. Phys. A: Math. Gen., On shooting methods for calculation of potential resonances 29 (1996) 6325-6342.

[86] E. Y. Sidky, I. Ben-Itzhak, Phase-amplitude method for calculating resonance energies and widths for one-dimensional potentials, Phys. Rev. A 60 (1999) 3586.

[87] Y. Huang, R. J. Le Roy, Potential energy, $\Lambda$ doubling and Born $\varnothing$ Oppenheimer breakdown functions for the $B{ }^{1} \Pi_{u}$ "barrier" state of $\mathrm{Li}_{2}, \mathrm{~J}$. Chem. Phys. 119 (2003) 7398.

[88] Y. Huang, R. J. Le Roy, Erratum: Potential energy, $\Lambda$ doubling and Born $₫$ Oppenheimer breakdown functions for the $B{ }^{1} \Pi_{u}$ "barrier" state of $\mathrm{Li}_{2}$, J. Chem. Phys. 126 (2007) 169904.

[89] A. U. Hazi, H. S. Taylor, Stabilization Method of Calculating Resonance Energies: Model Problem, Phys. Rev. A 1 (1970) 1109.

[90] J. Simons, Resonance state lifetimes from stabilization graphs, J. Chem. Phys. 75 (1981) 2465.

[91] R. Levebvre, Box Quantization and Resonance Determlnatlon: The Multichannel Case, J. Phys. Chem. 89 (1985) 4201-4206. 
[92] M. Garcia-Sucre, R. Levebvre, The use of the equivalence between boxing and scaling to determine resonance energies, Chem. Phys. Lett. 130 (1986) 240 .

[93] V. A. Mandelshtam, T. R. Ravuri, H. S. Taylor, Calculation of the Density of Resonance States Using the Stabilization Method, Phys. Rev. Lett. 70 (1993) 1932.

[94] F. Martín, Ionization and dissociation using B-splines: photoionization of the hydrogen molecule, J. Phys. B: At. Mol. Opt. Phys. 32 (1999) R197R231.

[95] H. Lefebvre-Brion, R. W. Field, The Spectra and Dynamics of Diatomic Molecules, Elsevier, 2004.

[96] J. M. Brown, A. S. C. Cheung, A. J. Merer, Lambda-Type Doubling Parameters for Molecules in Delta-Electronic States, J. Mol. Spectrosc. 124 (1987) 464-475, doi:10.1016/0022-2852(87)90157-3.

[97] S. P. Davis, M. C. Abrams, Sandalphon, J. W. Brault, M. L. P. Rao, Improved molecular parameters for the Ballik-Ramsay system of diatomic carbon $\left(b^{3} \Sigma_{g}^{-}-a^{3} \Pi_{u}\right)$, J. Opt. Soc. Am. B 5 (1988) 1838-1847.

[98] J. M. Brown, A. J. Merer, Lambda-Type Doubling Parameters for Molecules in П-Electronic States of Triplet and Higher Multiplicity, J. Mol. Spectrosc. 74 (1979) 488-494, doi:10.1016/0022-2852(79)90172-3.

[99] W. C. Richards, H. P. Trivedi, D. L. Cooper, Spin-orbit coupling in molecules, Clarendon Press, 1981.

[100] C. M. Marian, Spin-Orbit Coupling in Molecules, Rev. Comp. Chem. 17 (2001) 99.

[101] D. G. Fedorov, S. Koseki, M. W. Schmidt, M. S. Gordon, Spinфorbit coupling in molecules: chemistry beyond the adiabatic approximation, Int. Rev. Phys. Chem. 22 (2003) 551.

[102] L. Veseth, Spin-Orbit and Spin-Other-Orbit Interaction in Diatomic Molecules, Int. J. Quant. Chem. 18 (1970) 368.

[103] K. Pachucki, J. Komasa, Nonadiabatic corrections to the wave function and energy, J. Chem. Phys. 129 (2008) 034102.

[104] J. K. G. Watson, The inversion of diatomic Born-Oppenheimer-breakdown corrections, J. Mol. Spectrosc. 223 (2004) 39. 
[105] R. J. Le Roy, Improved parameterization for combined isotopomer analysis of diatomic spectra and its application to HF and DF, J. Mol. Spectrosc. 194 (1999) 189.

[106] R. M. Herman, J. F. Ogilvie, An effective Hamiltonian to treat adiabatic and nonadiabatic effects in the rotational and vibrational spectra of diatomic molecules, Adv. Chem. Phys. 103 (1998) 187.

[107] P. R. Bunker, R. E. Moss, The breakdown of the Born-Oppenheimer approximation: the effective vibration-rotation hamiltonian for a diatomic molecule, Molecular Physics 33 (1977) 417-424.

[108] R. J. Le Roy, Y. Y. Huang, Representing Born-Oppenheimer breakdown radial correction functions for diatomic molecules, J. Molec. Struct. (THEOCHEM) 591 (2002) 175-187, doi:10.1016/S0166-1280(02)002397.

[109] J. F. Ogilvie, J. Oddershede, S. P. A. Sauer, The rotational $g$ factor of diatomic molecules in state ${ }^{1} \Sigma^{+}$or $0^{+}$, Adv. Chem. Phys. 111 (2000) 475.

[110] H.-J. Werner, P. J. Knowles, G. Knizia, F. R. Manby, M. Schütz, et al., MOLPRO, version 2012.1, a package of ab initio programs, "see http://www.molpro.net", 2012.

[111] J. Tennyson, Vibration-rotation transition intensities from first principles, J. Mol. Spectrosc. 298 (2014) 1-6.

[112] E. Anderson, Z. Bai, C. Bischof, S. Blackford, J. Demmel, J. Dongarra, J. Du Croz, A. Greenbaum, S. Hammarling, A. McKenney, D. Sorensen, LAPACK Users' Guide, Society for Industrial and Applied Mathematics, Philadelphia, PA, third edn., ISBN 0-89871-447-8 (paperback), 1999.

[113] J. W. Demmel, O. A. Marques, B. N. Parlett, C. Vömel, Performance And Accuracy Of Lapack $₫$ s Symmetric Tridiagonal Eigensolvers, SIAM J. Sci. Comput. 30 (2008) 1508-1526.

[114] F. G. Van Zee, R. A. Van De Geijnand G. Quintana-Ortí, Restructuring the Tridiagonal and Bidiagonal QR Algorithms for Performance, ACM Trans. Math. Softw. 40 (2014) 18.

[115] G. Herzberg, Molecular Spectra and Molecular Structure. Volume I. Spectra or diatomic molecules, D. Van Nostrand Company, Inc, 1950. 
[116] M. Šimečková, D. Jacquemart, L. S. Rothman, R. R. Gamache, A. Goldman, Einstein A coefficients and statistical weights for molecular absorption transitions in the HITRAN database, J. Quant. Spectrosc. Radiat. Transf. 98 (2006) 130-155.

[117] J. Tennyson, K. Hulme, O. K. Naim, S. N. Yurchenko, Radiative lifetimes and cooling functions for astrophysically important molecules, J. Phys. B: At. Mol. Opt. Phys. .

[118] J. Tennyson, C. Hill, S. N. Yurchenko, Data structures for ExoMol: Molecular line lists for exoplanet and other atmospheres, in: $6^{\text {th }}$ international conference on atomic and molecular data and their applications ICAMDATA2012, vol. 1545 of AIP Conference Proceedings, AIP, New York, 186-195, 2013.

[119] J. Tennyson, S. N. Yurchenko, The ExoMol team, The ExoMol database: molecular line lists for exoplanet and other hot atmospheres, J. Mol. Spectrosc. .

[120] J. Dennis, R. Schnabel, Numerical Methods for Unconstrained Optimization and Nonlinear Equations, Society for Industrial and Applied Mathematics, doi:10.1137/1.9781611971200, URL http://epubs.siam.org/doi/abs/10.1137/1.9781611971200, 1996.

[121] A. N. Tikhonov, V. Y. Arsenin, Solutions of ill posed problems, Bull. Amer. Math. Soc. (N.S.) 1 (3) (1979) 521-524.

[122] S. N. Yurchenko, M. Carvajal, P. Jensen, F. Herregodts, T. R. Huet, Potential parameters of $\mathrm{PH} 3$ obtained by simultaneous fitting of ab initio data and experimental vibrational band origins, Contemp. Phys. 290 (2003) 59-67.

[123] S. N. Yurchenko, R. J. Barber, J. Tennyson, W. Thiel, P. Jensen, Towards efficient refinement of molecular potential energy surfaces: Ammonia as a case study, J. Mol. Spectrosc. 268 (2011) 123-129.

[124] M. Meuwly, J. M. Hutson, Morphing ab initio potentials: A systematic study of Ne-HF, J. Chem. Phys. 110 (1999) 8338-8347, doi: $10.1063 / 1.478744$.

[125] S. Skokov, K. A. Peterson, J. M. Bowman, Perturbative inversion of the $\mathrm{HOCl}$ potential energy surface via singular value decomposition, Chem. Phys. Lett. 312 (1999) 494-502, doi:10.1016/S0009-2614(99)00996-3. 
[126] J. L. Dunham, The energy levels of a rotating vibrator, Phys. Rev. 41 (1932) 721.

[127] G. Simons, R. Parr, J. Finlan, New alternative to Dunham potentials for diatomic-molecules, J. Chem. Phys. 59 (1973) 3229-3234, doi: $10.1063 / 1.1680464$.

[128] P. F. Fougere, R. K. Nesbet, Electronic structure of $C_{2}$, J. Chem. Phys. 44 (1966) 285.

[129] K. S. Sorbie, J. N. Murrell, Analytical Potentials for Triatomic-Molecules from Spectroscopic Data, Mol. Phys. 29 (1975) 1387-1407.

[130] L. Busevica, I. Klincare, O. Nikolayeva, M. Tamanis, R. Ferber, V. V. Meshkov, E. A. Pazyuk, , A. V.Stolyarov, Fourier transform spectroscopy and direct potential fit of a shelf-like state: Application to $E(4){ }^{1} \Sigma^{+} \mathrm{KCs}$, J. Chem. Phys. 134 (2011) 104307.

[131] H. Knockel, S. Ruhmann, , E. Tiemann, The $X^{1} \Sigma_{g}^{+}$ground state of $\mathrm{Mg}_{2}$ studied by Fourier-transform spectroscopy, J. Chem. Phys. 138 (2013) 094303.

[132] S. N. Huffaker, Diatomic molecules as perturbed Morse oscillators. I. Energy levels, J. Chem. Phys. 64 (1976) 3175-3181.

[133] S. N. Huffaker, Diatomic molecules as perturbed Morse oscillators. II. Extension to higher-order parameters, J. Chem. Phys. 64 (1976) 3175-3181.

[134] P. H. Dwivedi, S. N. Huffaker, Diatomic molecules as perturbed Morse oscillators. III. Perturbed eigenfunctions and Franck-Condon factors, J. Chem. Phys. 66 (1977) 1726-1735.

[135] S. N. Huffaker, P. H. Dwivedi, Diatomic molecules as perturbed Morse oscillators. IV. Franck-Condon factors for very high J, J. Chem. Phys. 69 (1978) 1303-1305.

[136] S. N. Huffaker, Diatomic molecules as perturbed Morse oscillators. V. Centrifugal-distortion, J. Chem. Phys. 72 (1980) 2601-2605.

[137] S. N. Huffaker, Diatomic molecules as perturbed Morse oscillators. VI. High-precision eigenfunctions, J. Chem. Phys. 74 (1981) 1217-1220.

[138] E. G. Lee, J. Y. Seto, T. Hirao, P. F. Bernath, R. J. Le Roy, FTIR emission spectra, molecular constants, and potential curve of ground state $\mathrm{GeO}, \mathrm{J}$. Mol. Spectrosc. 194 (1999) 197-202, doi:10.1006/jmsp.1998.7789. 
[139] R. J. Le Roy, Y. Huang, C. Jary, An accurate analytic potential function for ground-state $\mathrm{N}_{2}$ from a direct-potential-fit analysis of spectroscopic data, J. Chem. Phys. 125 (2006) 164310.

[140] A. A. Šurkus, R. J. Rakauskas, A. B. Bolotin, The Generalized PotentialEnergy Function for Diatomic-Molecules, Chem. Phys. Lett. 105 (1984) 291-294, doi:10.1016/0009-2614(84)85032-0.

[141] R. J. Le Roy, R. D. E. Henderson, A new potential function form incorporating extended long-range behaviour: application to ground-state $\mathrm{Ca}_{2}$, Mol. Phys. 105 (2007) 663-677.

[142] W. H. Press, S. A. Teukolsky, W. T. Vetterling, B. P. Flannery, Numerical Recipes - The Art of Scientific Computing, Cambridge University Press, 3rd edn., 2007.

[143] J. G. Herriot, C. H. Reinsch, Algorithm 600: translation of algorithm 507. procedures for quintic natural spline interpolation, ACM Trans. Math. Softw. 9 (1983) 258.

[144] J. G. Herriot, C. H. Reinsch, Algorithm 507: Procedures for Quintic Natural Spline Interpolation [E1], ACM Trans. Math. Softw. 2 (1976) 281.

[145] J. D. Poll, G. Karl, On vibrational frequencies of hydrogen molecule, Can. J. Phys. 44 (1966) 1467.

[146] R. J. Le Roy, R. B. Bernstein, Dissociation Energy and Vibrational Terms of Ground $\notin$ State $\left(X^{1}{ }^{1} \Sigma_{g}^{+}\right.$) Hydrogen, J. Chem. Phys. 49 (1968) 4312.

[147] L. Lodi, Theoretical Rotational-Vibrational Spectroscopy of Water, Ph.D. thesis, University College London, the thesis is available on-line at http://www.tampa.phys.ucl.ac.uk/ftp/eThesis/, 2008.

[148] R. J. Le Roy, R. B. Bernstein, Dissociation Energy and long-range potential of diatomic molecules from vibrational spacings of higher levels, J. Chem. Phys. 52 (1970) 3869.

[149] J. Goodisman, Dipole-Moment Function for Diatomic Molecules, J. Chem. Phys. 38 (1963) 2597-2599, doi:10.1063/1.1733557.

[150] A. J. Stone, input.F90 $\varnothing$ a Fortran90 module for parsing text input, see http://www-stone.ch.cam.ac.uk/programs/, 2005.

[151] S. N. Yurchenko, J. Tennyson, et al, ExoMol Molecular linelists - XVI. The spectrum of PH, PO and PS, Mon. Not. R. Astron. Soc. . 
[152] E. J. Barton, S. N. Yurchenko, J. Tennyson, ExoMol Molecular linelists - II. The ro-vibrational spectrum of SiO, Mon. Not. R. Astron. Soc. 434 (2013) 1469-1475. 